\title{
Franz-Xaver Kaufmann
}

\section{Steuerung wohlfahrtsstaatlicher Abläufe durch Recht}

Aufgabe des vorliegenden Beitrags ist es, die Funktion des Rechts im Kontext wohlfahrtsstaatlicher Interventionen genauer zu bestimmen. Dies setzt sowohl eine Präzisierung des Konzepts wohlfahrtsstaatlicher Intervention als auch eine steuerungstheoretische Perspektive voraus, die im vorliegenden Beitrag allerdings nicht im Detail entfaltet werden kann. Das Schwergewicht der Analyse liegt auf einer differenzierten Betrachtung von Sachverhalten, die in der bisherigen Diskussion mit dem Schlagwort der "Verrechtlichung der Sozialpolitik" allzu grobschlächtig erfaßt werden.

\section{Sozial- bzw. Wohlfahrtsstaatlichkeit}

Während in den 50er und 60er Jahren der nach dem Zweiten Weltkrieg im angelsächsischen Raum aufkommende Begriff des 'Welfare State' in Deutschland überwiegend kritisch-abwertend als 'Versorgungsstaat' aufgenommen wurde, hat der Begriff seit ca. 1980 als annäherndes Synonym zum eingeführten Begriff 'Sozialstaat' Eingang in das deutschsprachige sozialwissenschaftliche Schrifttum gefunden. Da "Sozialstaat" bisher weitgehend eine Domäne juristischer Interpretation geblieben ist, tun sich Sozialwissenschaftler offensichtlich leichter mit dem neu eingeführten Begriff und seinen diffuseren Bedeutungsüberschüssen.

Es herrscht weitgehende Übereinstimmung darüber, daß mit dem Begriff des Sozial- bzw. Wohlfahrtsstaats ein Staatstypus bezeichnet werden soll, der die Verantwortung der Gewährleistung menschenwürdiger Lebensbedingungen für alle ihm Angehörenden in expliziter Form übernimmt. ${ }^{1)}$ Was darunter jedoch im einzelnen zu verstehen ist, inwieweit der Staat durch solche Verantwortung zur Gewährleistung sozialer Teilhabe verpflichtet ist und welche Arten von Interventionen hierfür ohne nachteilige Nebenwirkungen geeignet sind, darüber sind die Auffassungen zwischen den politischen (und wissenschaftlichen!) Strömungen geteilt.

Nicht nur der Begriff des Wohlfahrtsstaats, auch derjenige des Sozialstaats leidet bisher an ungenügender sozialwissenschaftlicher Ausarbeitung; die nachfolgenden Ausführungen sind lediglich als Stichworte zu verstehen, in welche Richtung m.E. eine sozialwissenschaftliche Theorie des Wohlfahrtsstaats zu entwickeln wäre. 
Die Rede vom Sozial- oder Wohlfahrtsstaat ist mißverständlich: Sozialität ist nur ein Bestimmungsmerkmal des verfassungsmäßigen Staatskonzeptes (vgl. Art. 20 Abs. 1, 28 Abs. 1 Satz 1 GG), und ebenso kann 'Wohlfahrt' nur als ein Staatsziel unter anderen gelten. Es gibt auch kein einheitliches politisches Teilsystem, daß mit der Wahrnehmung der Wohlfahrtsfunktionen des Staates beauftragt wäre, wenngleich bestimmte Politikbereiche (z.B. Arbeits- und soziale Sicherungspolitik, Gesundheitspolitik, Familien- und Jugendpolitik, Bildungspolitik, Raumordnungspolitik) einen expliziteren Bezug zu wohlfahrtsstaatlichen Zielsetzungen als andere aufweisen. Während in der verfassungstheoretischen Diskussion das Verhältnis von Sozialstaatlichkeit und Rechtsstaatlichkeit stets als ein zum mindesten teilweise spannungsreiches gesehen wurde und somit die Wohlfahrtsziele stets in Relation zu anderen Staatsaufgaben gesetzt wurden, läßt die angelsächsische Explikation des Wohlfahrtsstaats eine solche Relativierung weitgehend vermissen. Die angelsächsische Wohlfahrtsstaatstheorie ist überwiegend schlicht normativ, d.h. sie setzt bestimmte Wohlfahrtsziele, ordnet ihnen bestimmte öffentliche Institutionen ("The Social Services") zu und vergleicht deren tatsächliche Entwicklung und Wirksamkeit mit den postulierten Zielsetzungen. Diese Verdinglichung des Konzepts hängt offensichtlich mit der wesentlich schwächeren Ausbildung des Staatskonzepts im angelsächsischen Raum zusammen (vgl. 2.1).

Die verfassungstheoretische Diskussion zur Sozialstaatlichkeit in der Bundesrepublik geht dagegen einen anderen Weg: Nachdem das Grundgesetz im Gegensatz zur Weimarer Reichsverfassung auf eine explizite Formulierung sozialer Grundrechte verzichtet hat, hat sich - nach anfänglicher Zurückhaltung hinsichtlich des materialen Gehalts der Sozialstaatsklauseln - eine an der Frage nach "sozialen Voraussetzungen der Realisierung grundrechtlicher Freiheit" (Böckenförde 1976: 238) orientierte Interpretation durchgesetzt. ${ }^{3)}$ Dies gestattet grundsätzlich eine überaus breite und offene Interpretation der Sozialstaatlichkeit, enthält dem Konzept aber alle konkrete Programmatik vor, wie sie in der meisten Länderverfassungen gegeben der Weimarer Reichsverfassung und der

Die inhaltliche Zurïen gegeben ist.

Sozialstaatlichkeit kann der grundlegenden Dimensionen menschlicher auf fehlenden Konsens hinsichtlich argument der verfassungstheoretischen Zurückhaltung ist verufen." tung, daß eine extensive Interpretation der Sozialstats vielmehr die Befürchi.S. subjektiver öffentlicher Sozialrechte die reztalstausel des Grundgesetzes Verfassungsnormen aushöhlen und die die rechtliche Verbindlichkeit der gesellschaftliche Akzeptanz der Verfassugreichsweise hohe politische und blik vermindern könnte.

Während die klassische, liberale Grundrechtsinterpretation in den Grund-
chten im wesentlichen Abwehrechte rechten im wesentlichen Abwehrrechte des Individuums gegen staatliche Eingrif- 
fe sah, neigt die sozialstaatlich orientierte Grundrechtsinterpretation dazu, staatliches Handeln als u.U. notwendige Voraussetzung ihrer faktischen Verwirklichkeitschancen zu postulieren. "Im Unterschied zur Abwehrkomponente verleiht diese Garantie i.d.R. aber keine subjektiven öffentlichen Rechte, sondern richtet sich in erster Linie an den Gesetzgeber und fordert ihn zu grundrechtsfundierender Tätigkeit auf. Nur dort, wo der Gesetzgeber selbst ein Minimum von Grundrechtsausstattung unterschreitet, sind aus dem objektivrechtlichen Gehalt der Grundrechte ausnahmsweise einklagbare Leistungsansprüche des einzelnen Grundrechtsträgers abgeleitet worden" (Grimm 1982: 57).

Damit werden Gemeinsamkeiten und Differenzen der deutschen sozialstaatlichen und der angelsächsischen wohlfahrtsstaatlichen Programmatik sichtbar: Gerade weil das deutsche Grundgesetz die Sozialstaatlichkeit explizit als geltenden Verfassungsauftrag an den Gesetzgeber betrachtet, muß sich die Verfassungsgerichtsbarkeit und die juristische Verfassungsinterpretation hinsichtlich ihrer inhaltlichen Ausgestaltung zurückhalten. Sozialstaatlichkeit erscheint hier ausdrücklich als Frage der Gesetzgebungspolitik, einer Politik allerdings, der die Verfassung verbindlich eine übergreifende allgemeine Richtung weist. Im angelsächsischen Raum fehlt es an einer vergleichbaren verfassungsrechtlichen Verfestigung des wohlfahrtsstaatlichen Gedankenguts. Es ist dort von vornherein stets lediglich ein politischer Akt, normative Forderungen wohlfahrtsstaatlich zu begründen. Mit der Verabschiedung des allgemeinen Teils eines Sozialgesetzbuches und den darin enthaltenen Zielsetzungen ( $\$ 1$ ) und spezifischen sozialen Rechten ( $\$ 3-10$ ) hat der Gesetzgeber in der Bundesrepublik überdies für Kernbereiche der Sozialpolitik (allerdings unter Ausschluß des Arbeitsrechts!) eine Konkretisierung und Systematisierung wohlfahrtsstaatlicher Grundsätze vorgenommen, der die angelsächsische Gesetzgebungspraxis nichts Vergleichbares zur Seite stellen kann."

Es ist daher nicht überraschend, daß in der angelsächsischen Diskussion der rechtlichen Fundierung wohlfahrtsstaatlicher Aktivitäten geringere Bedeutung zugemessen wird und statt dessen stärker auf die faktischen Leistungen, den hierfür erbrachten finanziellen, personellen und organisatorischen Aufwand sowie auch das Verhältnis von sozialen Problemen und möglichen oder wünschbaren Maßnahmen abgehoben wird. Ebenso wird nunmehr deutlich, weshalb die Problematik der 'Verrechtlichung', also die Frage nach möglichen Nebenwirkungen einer vorwiegend rechtlichen Steuerung wohlfahrtsstaatlicher Abläufe, vor allem im deutschen Kontext auftaucht, während die amerikanische Diskussion über 'Deregulation' vor allem die Wirtschaftspolitik betrifft.

Eine brauchbare sozialwissenschaftliche Theorie des Wohlfahrtsstaats muß versuchen, die pragmatische und die rechtliche Betrachtungsweise zu verbinden. Während die deutsche Tradition dazu neigt, die Differenz zwischen der rechtlichen Normierung und der Wirklichkeit des Sozialstaats zu minimieren, neigt 
die angelsächsische Diskussion dazu, die Bedeutung des unterschiedlichen Grades staatlicher Verpflichtung zur Erbringung sozialer Leistungen unterzubelichten. Beide Dimensionen entfalten jedoch ihre eigene Dynamik und Wirksamkeit, die für ein angemessenes Verständnis der Wirkungsweise wohlfahrtsstaatlicher Maßnahmen zusammen gedacht werden müssen.

Da die Schaffung zwingenden Rechts das spezifische Organisationsmittel des Staats ist, liegt die spezifische Differenz der Wohlfahrtsstaatlichkeit zu allen anderen Formen sozialen Engagements (beispielsweise von Kirchen, Gemeinden oder bestimmten gesellschaftlichen Gruppen) gerade in diesem Moment der rechtlichen Fundierung. Wohlfahrtsstaatlichkeit bestimmt sich demzufolge nach drei grundlegenden Gesichtspunkten:

1. Nach dem Ausmaß, in dem ein Staat explizite Verantwortung für die Gewährleistung menschenwürdiger Lebensbedingungen übernimmt;

2. nach dem Ausmaß, in dem diese Verantwortung nicht nur deklamatorischen Charakter trägt, sondern in eine Selbstbindung des Staates durch die Einräumung von Rechtsansprüchen an die Leistungsadressaten überführt worden ist;

3. nach dem Ausmaß, in dem konkrete staatliche Maßnahmen zur Gewährleistung der Erfüllung derartiger Rechtsansprüche ergriffen werden.

Die begriffliche Fixierung der Sozialstaatlichkeit durch Bezug auf staatliche Aktivität bedeutet selbstverständlich nicht, daß die Handlungen, welche im beitragen, ausschließlich von staatlichen Trägern und ihrem Personal ausgehen müssen. Es erscheint im Gegenteil gerade als Spezifikum der Sozialpolitik ${ }^{6}$, daß Status und Lebenslage von Individuen in erheblichem Maße von Einflüssen nicht-staatlicher Akteure abhängig sind. Staatliche Rechtssetzung und Finanzierung richten sich überwiegend nicht direkt an die zu Begünstigenden, sondern an Dritte, beispielsweise Wirtschaftsunternehmen, SozialversicherungsLeistungen das Ausmah Mehrstufigkeit wohlfahrtsstaatlich Teilhabe abhängig ist. Daraus resultiert eine fahrtsstaatlicher Interventitaatlicher Abläufe, die für die Steuerbarkeit wohl-

Vergegenwärtigen Intervention in seinen Grundzügen: Wüchst das Problem wohlfahrtstaatlicher wohlfahrtsstaatliche Programmün: Wie auch immer man im einzelnen die Beeinflussung sozialer Verhälik explizieren mag, stets bezieht sie sich auf die Möglichkeiten der Lebensfültnisse, von deren Eigenarten die alttäglichen mindestens Legitimation wohlfung von Individuen abhängig sind. Ziel oder oder Milderung individueller systematische Ort, an dem Not, Benachteiligung oder Beeinträchtigung. Der Maßnahmen entscheidet, ist dach der Erfolg oder Mißerfolg sozialpolitischer 
sozialen Kontext. Auf der anderen Seite ist am systematischen Ort des Ursprungs wohlfahrtsstaatlicher Maßnahmen der Gesetzgeber weder in der Lage, die vielschichtige Spezifität aller möglichen Einzelfälle zu berücksichtigen, noch kann er unmittelbare Hilfe gewähren. Die Zentralisierung der Entscheidungsbefugnisse erzwingt gleichzeitig die Generalisierung der Entscheidungsprämissen und die Notwendigkeit ihrer Spezifizierung in der Form von Einzelentscheidungen. In dem Maße, als der Staat (Bund, Land) bestimmte Handlungszusammenhänge gesetzlich regelt, folgt daraus zum mindesten deren Standardisierung und Vernetzung auf überregionaler Ebene, wenn nicht gar eine Vereinheitlichung und Zentralisierung.

Diesen Tendenzen zur Vereinheitlichung und Zentralisierung sind im Bereich wohlfahrtsstaatlicher Politik jedoch sachimmanente Grenzen gesetzt, d.h. es lassen sich Einflüsse und Zusammenhänge aufweisen, die die Wirksamkeit regulativer Politik in diesem Bereich in Frage stellen. Denn die sozialen Verhältnisse, auf die staatliches Handeln einwirken will, haben ihre eigene Struktur und Dynamik. Gerade der Umstand, daß Struktur und Dynamik der von Hegel so genannten 'Bürgerlichen Gesellschaft' entgegen ihren Verheißungen nicht die gleiche Freiheit Aller zu verbürgen vermochte, sondern neue Strukturen der Abhängigkeit hervorbrachte, hat ja bekanntlich zur Reaktivierung der bereits im merkantilistischen Staat offenkundigen politischen Bemühungen um staatliche Wohlfahrt geführt. ${ }^{8)}$ Das Einwirken des Staates auf die 'gesellschaftlichen Verhältnisse' ist kein einseitiger Vorgang, sondern ein Prozeß zunehmender Interdependenz zwischen den politischen und den übrigen, im Zuge der 'Trennung von Staat und bürgerlicher Gesellschaft' ausdifferenzierten gesellschaftlichen Teilsystemen.

Wohlfahrtsstaatliche Politik entfaltet sich typischerweise im Grenzbereich zwischen 'Staat' und 'bürgerlicher Gesellschaft', zwischen 'Öffentlichkeit' und 'Privatheit', und nur ein Teil der relevanten Aktivitäten erfolgt unmittelbar im Rahmen staatlicher Verwaltung. In hohem Umfange sind (zumeist hochorganisierte) Akteure beteiligt, die einer direkten staatlichen Weisungsbefugnis nicht unterliegen, deren Verhalten daher nur im Rahmen gesetzlicher Vorgaben zu kontrollieren ist, und die ihrerseits versuchen, auf die sie betreffenden rechtlichen Prämissen und praktischen Vollzüge staatlichen Handelns i.S. ihrer eigenen Interessen Einfluß zu gewinnen. Hierbei kann es sich entweder um (häufig verbanc'smäßig organisierte) gesellschaftliche Akteure handeln ${ }^{\text {9) }}$ oder aber um unter staatlichem Einfluß gegründete, jedoch formal unabhängige Organisationen öffentlich- oder privatrechtlichen Charakters (vgl. Schuppert 1981, Gessner/Winter 1982, Hood 1986 a).

Mehr noch: Selbst im Bereich der unmittelbaren Staatsverwaltung und zumal beim Vorliegen föderalistischer Strukturen sind (personelle und organisatorische) Eigeninteressen zu vermuten, die - bezogen auf zentralstaatliche Intentionen zu Zielverschiebungen und Implementationswiderständen führen können (klas- 
sisch hierzu Pressman und Wildavsky 1973, zusammenfassend Hood 1976, für Deutschland Scharpf, Reissert, Schnabel 1976, 1978). Das gilt auch und gerade in Fällen, wo der Gesetzgeber die Mitwirkung anderer politischer Akteure vorschreibt. So bemerkt Lehner (1979: 621), "daß die Mischfinanzierung eine Politiksteuerung durch Parlamente oder politisch verantwortliche exekutive Instanzen kaum zuläßt. Probleme und Problemlösungen müssen vielmehr möglichst exklusiv in den spezialisierten Interaktionen der Politikverflechtung definiert und ausgehandelt werden. Die an diesen Interaktionen beteiligten Fachbürokratien des Bundes und der Länder können und müssen also selbst als politische 'Entrepreneure' auftreten und die Politiksteuerung übernehmen".

Zusammenfassend läßt sich festhalten, daß unter Bedingungen einer politischen Verfassung, die Herrschaft demokratischer Legitimation unterstellt und eine explizite Verantwortung der Regierung für Dimensionen individueller und kollektiver Wohlfahrt postuliert, staatliches Handeln nur über eine beschränkte Autonomie verfügt und in erheblichem Umfang auf das Zusammenwirken mit anderen - parastaatlichen und gesellschaftlichen - Akteuren angewiesen ist. In steuerungstheoretischer Hinsicht ist es sogar problematisch, Staatlichkeit als ein einheitliches Handlungszentrum zu begreifen. Selbst die politischen Kernstrukturen sind - schon aufgrund zunehmender Komplexitätssteigerung des politischen Systems selbst - nur als eine Vielzahl von Akteuren angemessen zu begreifen. Die Steuerungsproblematik im Sozial- oder Wohlfahrtsstaat kann
daher zunächst in einem daher zunächst in einem sehr allgemeinen Sinne als das Problem der Koordination dieser Vielzahl beteiligter Akteure durch staatliche Vorgaben bestimmt
werden.

Endlich kann keineswegs vorausgesetzt werden, daß wohlfahrtsorientierte Interventionen des Staates hinsichtlich ihrer Ausgestaltung und ihres Operationsmodus auch bei erfolgreicher Koordination der beteiligten Akteure den Sozialpolitische Interventionen sind typischerwe die sie zu lösen beanspruchen. zeichnet, daß Normadressaten und typischerweise durch den Umstand gekennergeben sich spezifische Probleme, Normbenefiziare nicht identisch sind. Hieraus

Das Problem wohlfahrtsstaatlicher Interckzukommen sein wird.

verdichten, ob und unter welchen Bedervention läßt sich somit zur Frage Verfügung stehenden Mitteln in den Bedingungen der Staat mit den ihm zur Programmatik verbundenen Versprechungen einzulösen der wohlfahrtsstaatlichen tierenden Ansprüche zu befriedigechungen einzulösen bzw. die daraus resulProblem der Bewirkbarkeit bedingungen, bei denen eine 'Brechung' der politischen Interventionen durch die priori zu vermuten sind (10) Dieses Problem vorliegenden Zusammenhang ausschließlich in zahlreiche Aspekte, die im ten und Grenzen rechtlich konditionierter Stich der Perspektive der Möglichkei- 


\section{Zur Funktion des Rechts im Kontext sozialer Steuerung}

Nach herkömmlicher Auffassung (zumal in Deutschland) besteht ein sehr enger Zusammenhang zwischen Staat und Recht, der in der Idee der Rechtsstaatlichkeit auf den Begriff gebracht wird. Das Staatswesen und seine Organe werden selbst durch Recht konstituiert, und alle Staatstätigkeit findet ihre Möglichkeiten und Grenzen durch Recht geregelt. Gleichzeitig setzt staatliches Recht eine Rahmenordnung für die Entfaltung der 'freien gesellschaftlichen Kräfte', deren Verletzung durch Mobilisierung staatlicher Sanktionsgewalt in Grenzen gehalten wird. In dem Maße jedoch, als dem Staate nicht mehr nur Sicherheits- und Ordnungsfunktionen, sondern auch sozial gestaltende Funktionen - insbesondere solche der Beeinflussung der wirtschaftlichen Entwicklung, der Daseinsvorsorge und der Gewährleistung sozialer Teilhabe - zugemutet bzw. von seinen Organen übernommen werden, ändert sich die Funktion des Rechts in wesentlichen Dimensionen. Dies ist in der deutschen Staatsrechtslehre vor allem im Gefolge Forsthoffs früh erkannt, jedoch bis heute nicht systematisch bewältigt worden.

Es ist jedoch zu vermuten, daß die interdisziplinäre Öffnung der Rechtswissenschaft zu den Sozialwissenschaften gerade mit diesem Funktionswandel nachhaltig zusammenhängt (vgl. Grimm 1982, kritisch Blankenburg/Treiber 1982). In dem Maße, als staatliches Recht mit Bezug auf die gesellschaftlichen Verhältnisse nicht mehr nur eine Rahmenordnung - als öffentlich sanktioniertes System privater Rechtsverhältnisse und als Katalog verbotener Verhaltensweisen - vorgibt, sondern beansprucht, in diese Verhältnisse i.S. materialer Ziele steuernd einzugreifen, insoweit also das Ziel staatlicher Interventionen nicht mehr bloß die Verhinderung, sondern die Bewirkung bestimmter sozialer Verhaltensweisen betrifft, bedarf die Rechtsgestaltung solider Kenntnisse der Zusammenhänge und der in ihnen wirksamen Kräfte. Im Rahmen einer Tagung über "Gesetzgebungstheorie und Rechtspolitik" führt das gestellte Thema daher zur Frage an den Sozialwissenschaftler, welches aus seiner Sicht die Möglichkeiten und Grenzen staatlichen Eingreifens mit Hilfe von Recht zur Beeinflussung sozialer Problemlagen und zur Gewährleistung sozialer Teilhabe sind.

Obwohl (oder gerade weil) Soziologen (zumal in Deutschland) abstrakte Fragestellungen dieses Typs lieben, verführt die Frage leicht zu einer für die Gesetzgebungstheorie wenig hilfreichen, nämlich sehr allgemeinen Antwort. Als Beispiel kann auf den - im übrigen ausgezeichneten - Beitrag von Teubner (1984) verwiesen werden. Im Anschluß an die jüngste Version systemtheoretisehep Denkens, die Theorie selbstreferentieller bzw. autopoietischer Systeme11), wird als Bedingung regulatorischer Rechtsanwendung eine "'strukturelle Kupplung' von Politik, Recht und gesellschaftlichem Lebensbereich" (Teubner 1984: 316) postuliert, ein in seiner Allgemeinheit durchaus plausibles und das Verwirrspiel der jüngsten "Verrechtlichungsdebatte" (etwa Voigt (Hg.), 
1980, 1983a,b) in bemerkenswerter Weise auflösendes Kriterium. Es kann jedoch für die Gesetzgebungtheorie nur brauchbar gemacht werden, wenn es gelingt, diese Vorstellung in einer Weise zu konkretisieren, daß die spezifischen Differenzen typischer Problemlagen sichtbar werden, welche den verschiedenen Möglichkeiten politischer Steuerung mittels Recht als mehr oder weniger zugänglich erscheinen.

\subsection{Dimensionen der Verrechtlichung in steuerungsanalytischer Perspektive}

Die Problemformel 'Steuerung durch Recht' ist - ebenso wie die jüngst hochgespielte der 'Verrechtlichung' - zu abstrakt, um analytisch brauchbar zu sein. Da beide Formeln auf denselben Problemhorizont verweisen, sei zunächst versucht, den Erscheinungsraum des Rechts in einer für steuerungsanalytische Zwecke geeigneten Form zu dimensionieren. ${ }^{12)}$

Rechtsphänomene sind bekanntlich wesentlich älter als die Entstehung des modernen Staates, doch geht mit seiner Entstehung eine Qualitätsänderung des Rechts, seine 'Positivierung', d.h. gleichzeitig 'Verstaatlichung' und 'Vergesetzlichung', einher. Diese Form der Verrechtlichung (hier i.S. von Habermas 1981: $524 \mathrm{ff}$.) hat sich auf der Basis des römischen Rechts in den kontinentaleuropäischen Staaten wesentlich stärker entwickelt als in den Ländern des 'Common Law', deren Staatsauffassung auch wesentlich weniger institutionell geprägt ist (vgl. Nettl 1968, Dyson 1982). ${ }^{13\}}$ Dementsprechend ist sowohl der hoheitliche Charakter der Rechtssetzung und Rechtsdurchsetzung als auch der Anspruch auf ein möglichst widerspruchsfreies Rechtssystem in Kontinentaleuropa wesentlich ausgeprägter - und ebenso die Hoffnungen, die in einen 'starken Staat' gesetzt werden. Und in der Tat hat - insbesondere in Preußen und Frankreich - bereits der merkantilistische Staat sich eine stabile organisationelle (öffentliche Verwaltung) und personelle (Beamtenschaft) Infrastruktur geschaffen, die bis heute eine vergleichsweise höhere staatliche Steuerungskapa-
zität auf dem Kontinent vermuten lassen.

Die deutsche 'Verrechtlichungsdebatte' steht im Horizont der Vorstellungen an das von ihm selbst gesetzte Recht gebunden ist. Während unter den liberalen Prämissen einer möglichst weitgehenden Entkoppelung von Staat und bürgerFunktionen zugesproch Recht - soweit ihm überhaupt verhaltenssteuernde ihren auf Sicherheit und Ordnung - sich an die Organe des Staates selbst und sich das Aufgabenspektrum Ordnung beschränkten Aufgabenbereich richtete, weitet den Prämissen der Worm und damit auch der Kreis der Normadressaten unter Begrenzbare aus. Die Grfahrtsstaatlichkeit ins kaum mehr rechtsimmanent - und damit je nach vorhen erscheinen zunächst lediglich politischer Natur 
Situation wird die Frage nach den Handlungspotentialen oder Steuerungsmöglichkeiten des Staates sowie ihren Grenzen dringlich: Ansprüche an das Tätigwerden des Staates können nur noch rational unter dem Gesichtspunkt der Erfolgsaussichten und Nebenwirkungen solchen Tätigwerdens erörtert werden, insoweit als die verfassungsmäßigen Grenzen fließend geworden sind. ${ }^{14)}$

Die Verrechtlichungsdebatte stellt eine spezifische Version der Diskussion um 'Staatsversagen' dar (so auch Voigt 1983b: $23 \mathrm{ff}$.), ein Begriff aus der steuerungstheoretischen Debatte (s.u.). Sie bleibt jedoch hinter der steuerungsanalytischen Fragestellung insofern zurück, als sie dem Recht selbst steuernde Wirkungen zumutet, obwohl dieses, wie zu zeigen sein wird, nur eine Teilfunktion sozialer Steuerung übernehmen kann. Das gilt selbst für den im Bereich der öffentlichen Verwaltung dominierenden Typus der hierarchischen Steuerung. Überdies haben die meisten Steuerungsformen rechtliche Grundlagen, die allerdings mehr oder weniger in den Vordergrund rücken können.

In einem sehr allgemeinen Sinne kann von 'Verrechtlichung' überall dort gesprochen werden, wo die soziale Bedeutung des (geschriebenen) Rechts zunimmt. Mit dieser Bestimmung ist allerdings erkenntnismäßig wenig gewonnen. Wie Teubner (1984: 291) zu Recht bemerkt, ist "in der aktuellen Diskussion der Begriff Verrechtlichung dermaßen inflationiert, daß man ihn sorgfältig eingrenzen muß, um überhaupt sinnvolle Aussagen machen zu können”. Verrechtlichung in diesem allgemeinen Sinne läuft auf verschiedenen Ebenen ab, die - teilweise im Anschluß an Voigt (1981) und Zacher (1984) - wie folgt unterschieden werden können:

\section{Verstaatlichung:}

Der wachsende Einfluß des Staates auf die Gestaltung und Systematisierung des Rechts, insbesondere die Zunahme zentral-staatlicher Kompetenzen zur rechtlichen Gestaltung gesellschaftlicher Lebensbereiche. ${ }^{15)}$ Dem Prozeß der Verstaatlichung des Rechts als Kompetenzverlagerung entspricht in steuerungstheoretischer Perspektive der Prozeß der Zentralisierung von politischen Entscheidungen.

2. Vergesetzlichung:

Die Standardisierung, Generalisierung, Systematisierung und Differenzierung des Rechts auf der Basis legislativer Akte. Vorstaatliches Recht (und auch das 'Case Law') ist typischerweise an Brauch und Sitte sowie an richterliche Einzelentscheidungen gebunden. Mit der Positivierung des Rechts vollzieht sich - wie Luhmann (1972: 207 ff.) hervorhebt - nicht nur eine stärkere Systematisierung und Differenzierung, sondern vor allem auch die Institutionalisierung einer Änderbarkeit des Rechts: "Das historisch Neue und Riskante der Positivität des Rechts ist die Legalisierung von Rechtsänderungen" (209). Insofern ist die Vergesetzlichung eine 
notwendige Reabedingung für eine politische Manipulierbarkeit des Rechts und damit für interventionistische Politik.

3. Die Verrechtlichung sozjaler Beziehungen:

Von einer sozialen 'Steuerung durch Recht' kann sinnvollerweise nur don gesprochen werden, wo Handlungsweisen oder soziale Interaktionen 'verrechtlicht' sind, d.h. in an der rechtlichen Normierung orientierter Weise ablaufen ( $\mathrm{vgl}$. Ellscheid 1979, Kaufmann 1984a). Mit der 'Berufung auf das Recht' geht typischerweise eine Qualitätsänderung sozialer Beziehungen einher, die andere Formen sozialer Steuerung - etwa 'Kollegialität' oder 'Solidarität' - in Frage stellen.

4. Bürokratisierung: ${ }^{16)}$

Der Ausbau der öffentlichen Verwaltung als explizit rechtsbezogener Organisationsform sowie allgemeiner die Verrechtlichung von Beziehungen innerhalb und zwischen formalen Organisaionen. Hiermit geht regelmäßig einher eine weitere Differenzierung und Verdichtung der gesetzlichen Normen und damit häufig eine Einschränkung der Handlungs- und Ermessensspielräume nachgeordneter Stellen.

5. Justizialisierung:

Die Tendenz, soziale Konflikte nicht nur zu verrechtlichen, sondern sie richterlicher Entscheidung zu unterwerfen. Diese Tendenz ist für verschiedene Rechtsgebiete sehr unterschiedlich ausgeprägt, häufig werden andere Konfliktregelungsformen (z.B. Kontaktabbruch, fortgesetztes Verhandeln, Schiedsgerichtsbarkeit, Schlichtung) vorgezogen. Justizialisierung, die auch unter den Bedingungen vorstaatlichen Rechts möglich ist, entfaltet beim gleichzeitigen Vorliegen der übrigen Verrechtlichungstendenzen eine diese verstärkende Wirkung, und zwar insbesondere auf den Ebenen 2 bis 4.

Auch wenn die fünf skizzierten Teilprozesse sich tendenziell wechselseitig verstärken und insofern zu Recht von einem Gesamtphänomen 'Verrechtlichung' lichung und ihren Folgen doch regelmäßig auf partielle Zusammenhänge, die ebenen anzusiedeln sind. ${ }^{17}$ ) Grenzbereich zwischen zwei benachbarten Analyse-

Von einer sozialen 'Steuerung durch Recht' kann sinnvollerweise nur dort gesprochen werden, wo Handlungsweisen oder soziale Interaktionen 'verrechtlicht' sind, d.h. in an der rechtlichen Normierung orientierter Weise ablaufen, (mit-)bewirkt wird. wissenschaftliche Perspektive. Perspektive in zweierlei Hinsicht: unterscheidet sich von der juristischen mierung selbst, die Vergesetzt: Nicht der Tatbestand der rechtlichen NorOrientierung an Rechtsnormen. Diese, ist entscheidend, sondern die soziale 
konform zu sein: Auch die bewußte Gesetzesumgehung oder (z.B. protestierende) Rechtsverletzung ist an der rechtlichen Normierung orientiertes Handeln, wenngleich sie i.d.R. nicht zu stabilen Handlungsketten zwischen mehreren Akteuren führt. In der politikwissenschaftlichen Perspektive erscheint 'Steuerung durch Recht' als 'regulative Politik', d.h. als Intervention des Staates mit Hilfe von Geboten und Verboten (vgl. Mayntz 1983a, Hucke 1983), unabhängig vom Erfolg bzw. der Rechtswahrnehmung durch die Normadressaten. Die politikwissenschaftliche Perspektive setzt die hoheitliche Steuerungskompetenz des Staates zum mindesten heuristisch voraus und analysiert sodann die Erfolgs- und Mißerfolgsbedingungen. ${ }^{18)}$

Offensichtlich liegen diesen drei Perspektiven unterschiedliche Ausgangspunkte, und d.h. auch implizite normative Annahmen zugrunde: Für den Juristen ist die objektive Existenz des Rechts als Ordnungsmacht, für den Politikwissenschaftler die Fähigkeit des Staates, Politik zu betreiben, für den steuerungstheoretisch orientierten Soziologen dagegen das Gelingen der Koordination von Handlungen verschiedener Akteure unter präsumtiven übergeordneten Gesichtspunkten $^{19)}$ Ausgangspunkt aller weiteren Überlegungen. Diese drei Perspektiven sind m.E. nicht nur kompatibel, sondern mit Bezug auf das Problem "Steuerung durch Recht" komplementär.

\subsection{Dimensionen sozialer Steuerung}

Herkömmliche Diskussionen um politische und gesellschaftliche Steuerung gehen meist davon aus, daß das politische und das ökonomische System unterschiedlichen, jedoch für sich einheitlichen Steuerungsimperativen unterliegen. Die Verbalschlachten 'Marktwirtschaft versus Staat' oder 'Markt versus Plan' stellen jedoch eine nur noch politisch verstehbare Verschleierung der faktischen Steuerungsprobleme dar. Zum einen lassen sich die als spezifisch behaupteten Steuerungselemente wie Macht/Recht und Hierarchie einerseits, Geld und Markt andererseits in beiden Bereichen nachweisen, und zum anderen stellen die darauf bezogenen Steuerungstypen keine erschöpfende Beschreibung der Formen der Handlungskoordination im politischen und ökonomischen und erst recht nicht in den übrigen Gesellschaftsbereichen dar.

Bezugsproblem einer soziologischen Steuerungstheorie ist die Erklärung des Zustandekommens von Handlungsketten, d.h. des sozialen Tatbestandes, daß wie alltäglich, aber auch in größeren Zusammenhängen zu beobachten spezifische Handlungen verschiedener Akteure in im Regelfall erwartbarer Weise spezifische Anschlußhandlungen nach sich ziehen. ${ }^{20)}$ Im Zuge fortschreitender Pazifizierung größerer Räume und gesellschaftlicher Arbeitsteilung konnten immer längere Handlungsketten stabilisiert werden (vgl. Elias 1976: $321 \mathrm{ff}$.) und genau dies erscheint als Bedingung der spezifischen Leistungsfähig- 
keit moderner Gesellschaften. Die im Regelfall erwartbare Verkettung von Handlungen prinzipiell als unabhängig zu denkender Akteure setzt jedoch erfolgreiche Handlungskoordination voraus, und wie, d.h. in welchen verschiedenen Formen dies geschehen kann, ist die Ausgangsfrage des hier vertretenen steuerungstheoretischen Ansatzes.

Erfolgreiche Handlungskoordination setzt aus steuerungsanalytischer Sicht die Lösung von drei Problemen voraus:

1. Das Problem der Bestimmung oder Abstimmung von Kriterien 'richtigen' Handelns für oder zwischen beteiligten Akteuren (Guidance). Handlungskoordination setzt typischerweise Richtwerte oder Zeichen voraus, an denen die Akteure die erwartete Nützlichkeit oder Schädlichkeit ihres Handelns in einem größeren, über die Interaktionspartner hinausreichenden Zusammenhang beurteilen können. Offensichtlich kommt dem Recht gerade zur Lösung dieses Problems entscheidende Bedeutung zu, doch können ähnliche Effekte z.B. auch durch die Prozesse der Preisbildung oder - in dauerhaften Interaktionszusammenhängen - durch Bildung von Sitte und Tradition
erreicht werden.

2. Das Problem der Gewährleistung 'einsichtigen', d.h. norm- und situationsangemessenen Verhaltens (Control). Die Lösung dieses Problems hat sowohl informationelle wie motivationale Aspekte: Zum einen geht es bei den beteiligume der Handlungskoordination förderliche Situationswahrnehmung etwa durch die Definition von stimulieren, am Beispiel des Rechts also Zum anderen geht es aber auch darum, die d.h. den Erhaltungsbedingungen des größeren kompatiblem bzw. förderlichen Verhalten zu motivieren. In dieser Beziehung kommt dem Recht meist nur eine subsidiäre Funktion zu: Es vermag zwar die Nachteile, welche aus einem nicht normkonformen Verhalten erwachsen, näher zu bestimmen, hat jedoch i.d.R. auf die Gratifikationen
der Normkonformität nur einen geringen Einfluß. ${ }^{21}$ )
Das Problem der Sicher

3. Das Problem der Sicherung von Kommunikation über Ergebnisbewertungen als Voraussetzung des Lernens der beteiligten Akteure (Evaluation). Im allem die Steuerung durch Recht dient der Lösung dieses Problems vor nen etwa auf die der Gerichte, während im Bereich von Organisatiobeispielhaft hinzuweisen ist.

Aus diesen drei Problemstellungen lassen sich die Hauptdimensionen bzw. Teilfunktionen dauerhafte 
Die Koordination sozialer Handlungen ist nie 'mechanisch' gesichert, da Handlungen regelmäßig Ressourcen der Handelnden beanspruchen. Wesentliche Merkmale steuerungstheoretisch relevanter Handlungsketten sind daher:

- Die Wiederholbarkeit von Handlungen im Zeitablauf,

- die Existenz eines Initiierungs- und Erhaltungsaufwands bei Handlungsketten (ökonomisch: Transaktionskosten),

- Steigerungsmöglichkeiten der Leistungsfähigkeit bei der Verkettung von Handlungen infolge von Lernprozessen.

Während im Rahmen kurzer Handlungsketten die Probleme der Handlungsnormierung, -kontrolle und -beurteilung u.U. ausschließlich interaktiv gelöst werden können (operationale Steuerung) setzt die Etablierung längerer Handlungsketten regelmäßig zusätzliche Vorkehrungen voraus, die mit dem Begriff der institutionellen Steuerung angesprochen werden. Es lassen sich verschiedene institutionelle Arrangements aufweisen, die unter bestimmten Bedingungen erfolgreiche Handlungskoordination wahrscheinlich machen. Unter institutionellen Arrangements seien dabei Konfigurationen von Normen (Rules) - im weitesten Sinne, unter Einschluß von z.B. Definitionen, Routinen und Handlungsmaximen - verstanden, die in einem bestimmten Handlungskontext (action arena) im Regelfall als Handlungsprämissen wirken. Steuerungstheoretisch relevant ist nun die Abstraktion der Bedingungen, unter denen derartige institutionelle Arrangements erfolgreiche Handlungskoordination wahrscheinlich machen. Derartige Bedingungen beziehen sich z.B. auf Zutritt, Sachbereich, Positionen, Kompetenzen, Informationsformen, die Gewichtung von Einfluß bei Entscheidungen oder die Verteilung von Kosten und Nutzen (vgl. E. Ostrom, 1986: 468 ff.).

Aus der sozial- und wirtschaftswissenschaftlichen Literatur sind zahlreiche Konfigurationen bekannt, denen steuernde Qualitäten zugesprochen werden. Ihre Leistungsfähigkeit ist unter dem Gesichtspunkt der Lösung der drei oben genannten genannten Bezugsprobleme der Handlungskoordination zu prüfen. Auf diese Weise lassen sich 'reine Typen sozialer Steuerung' analytisch entwikkeln, die sich in charakteristischen Merkmalen sowohl ihrer Regelstruktur als auch der Bedingungen ihrer koordinativen Wirksamkeit unterscheiden. In diesem Sinne wurden bisher Markt (Krüsselberg 1986, Gretschmann 1986, E. Ostrom 1986), Hierachie (Krüsselberg 1986, Hegner 1986), Solidarität (Kaufmann 1984b, Gretschmann 1986, Hegner 1986), Wahlsysteme (Herzberg und V. Ostrom 1986) sowie Kollegialität (Majone 1986) untersucht. Ein Großteil der faktisch ablaufenden Prozesse komplexer Handlungkoordination - man denke etwa an die Entwicklung einer Raumfähre oder die Durchsetzung schadstoffarmen Treibstoffs - ist allerdings nur als Ergebnis des Zusammenspiels von Elementen unterschiedlicher Steuerungsformen angemessen zu begreifen. 


\subsection{Recht - steuerungstheoretisch}

Welche Bedeutung hat nun das staatlich gesetzte Recht für die Koordination von Handlungen? Diese Frage bedarf einer differenzierten und vielschichtigen Antwort, die hier nur in ihren für die Erklärung der Steuerung wohlfahrtsstaatlicher Abläufe wichtigen Dimensionen angedeutet werden kann.

Aus steuerungstheoretischer Perspektive ist zunächst festzuhalten, daß für die individuelle Handlungskoordination, also die Sequenzierung von Handlungen eines Individuums, das ja an einer Vielzahl von Handlungsketten partizipiert, Rechtsnormen typischerweise nur eine sehr indirekte Rolle spielen. ${ }^{\text {3) }}$ Dagegen ist es offenkundig, daß die Handlungskoordination zwischen verschiedenen Akteuren fast immer eine (zumindest latente) rechtliche Komponente hat, auf die man sich - spätestens im Notfall - berufen kann. Diese kann jedoch sehr unterschiedlich ausgestaltet sein. Für unseren sozialpolitischen Zusammenhang sind vor allem folgende Typen rechtlicher Normierung bedeutungsvoll:

1. Verbote: Neben den hier meist nur indirekt relevanten strafrechtlichen Normen sind vor allem Verbote zu nennen, die die Benachteiligung sozial schwächerer Personen (Gruppen) verhindern sollen, beispielsweise im Arbeitsvertrags- oder Mietrecht.

2. Gebote: Hierzu gehören neben den verschiedenen, die Finanzierung wohlfahrtsstaatlicher Aktivitäten erst ermöglichenden Abgabepflichten vor allem Sicherheitsnormen und Mindeststandards (z.B. im Arbeitsschutz, Umweltschutz oder Baurecht), aber auch Berichts- oder Beteiligungspflichten (z.B. Mitbestimmung, Sozialrecht).

3. Einräumung von Rechtsansprüchen: Diese können sich auf bestimmte Leistungen oder Unterlassungen Dritter, häufig aber auch auf Mitwirkungsmöglichkeiten beziehen. Von Rechtsansprüchen sei in diesem Zusammenhang nur insoweit die Rede, als dem Normbenefiziar die rechtlich anerkannte Möglichkeit eingeräumt wird, zur Realisierung seines Rechtsanspruches gegenüber den Normadressaten hierfür zuständige

4. Formulierung von $Z$ wecken Aufsichtsämter usw.) zu bemühen.

hiermit keine greifende Gesichtspunkte erzwingbaren Normen gesetzt, sondern überhalten und die Richtung der Handliert, die für die Beurteilung von Sachverkontext maßgeblich sein sollen.

5. Definition

nung befaßt von Bedingungen: Der ganz überwiegende Teil der Rechtsordstimmte Verhaltensweiser Definition von Bedingungen, unter denen betion von Maß, Gewicht und Wöglich sein sollen. Dies geht von der Defini- 
bis zu den Bedingungen gerichtlicher oder administrativer Gewährungen bzw. Versagungen.

6. Regelung von Verfahren: Die Entwicklung einer Normkonfiguration, die meist auch Normen nach Ziff. 1 bis 5 enthält und bedingte Handlungsverkettungen von mindestens zwei beteiligten Akteuren programmiert. Von Bedeutung sind in unserem Zusammenhang nicht nur Verwaltungs- und Gerichtsverfahren, sondern auch die Regelung von Verhandlungssituationen, etwa im Unternehmens- und Arbeitsrecht, aber z.B. auch im Bereich des Gesundheitswesens.

7. Errichtung von Einrichtungen öffentlichrechtlicher oder privatrechtlicher Art. Auf diese Weise entstehen neue, zum mindesten formal unabhängige Akteure, denen bestimmte Aufgaben zugewiesen werden können. Gerade im Bereich der Daseinsvorsorge wird gerne von einer solchen "Auslagerung öffentlicher Aufgaben" Gebrauch gemacht. Häufig wird dabei gleichzeitig der Einfluß staatlicher und nicht-staatlicher Akteure institutionalisiert (z.B. Sozialversicherungsträger, Energiewirtschaft).

8. Normierung staatsinterner Koordination: Hierzu gehören insbesondere das staatliche Organisationsrecht und das sog. instrumentelle Verwaltungsrecht, also der rechtliche Kern der institutionellen Steuerung der staatlichen Akteure. Im Unterschied zu den vorangehenden Normierungstypen begründen diese Rechtsnormen typischerweise keine direkten Mitwirkungsmöglichkeiten nicht-staatlicher Akteure, sondern regeln die Handlungsmöglichkeiten staatlicher Akteure. Dennoch kann ihre Ausgestaltung von erheblicher Bedeutung für die Wahrscheinlichkeit von Anschlußhandlungen derartiger Akteure sein.

Staatliche Interventionen mittels Rechtssetzung bedienen sich typischerweise unterschiedlicher Kombinationen dieser Normierungstypen, und im Regelfall beinhalten staatliche Interventionen nicht ausschließlich Rechtssetzungsakte, diese bedürfen zu ihrer Wirksamkeit vielmehr zusätzlicher Maßnahmen: Etwa der Schaffung neuer Verwaltungsabteilungen bzw. Behörden als Ausführungs- oder Kontrollorgane, die Bereitstellung von Finanzmitteln zur Subventionierung nicht-staatlicher Träger oder zur Erfüllung eingegangener Verpflichtungen, usw.

$\mathrm{Ob}$ und unter welchen Bedingungen "Recht und Geld: Die Wirkungsmittel des Wohlfahrtsstaates" (Luhmann) tatsächlich geeignet sind, bestimmte Politikziele zu erreichen bzw. bestimmte Probleme zu lösen, bleibt umstritten. In der hier vertretenen steuerungstheoretischen Perspektive dient Recht primär der Handlungsnormierung, Geld der Handlungskontrolle. Zwar sind die Normkonfigurationen im Rahmen institutioneller Koordination nie ausschließlich rechtlicher Art, doch spielen Rechtsnormen - vor allem im Grenzbereich von Staat und 'Gesellschaft' - regelmäßig eine konstitutive, häufig auch eine indikative Rolle. Geldleistungen dagegen kommt erhebliche motivierende Kraft zu, um erwünsch- 
te Anschlußhandlungen zu bewirken, zum mindesten wenn ihre Vergabe an bestimmte Bedingungen gebunden wird.

Allerdings bleiben Recht und Geld im Regelfall mehrstufiger Politikverwirklichung zu unspezifisch, um zweckmäßige Anschlußhandlungen sicherzustellen. Sie vermögen nicht eine angemessene Situationswahrnehmung und adäquate Handlungskompetenzen der Normadressaten sicherzustellen. Recht und Geld vermitteln als Kommunikationsmedien "Entscheidungsprämissen und können dadurch die Bindungswirkung von Entscheidungen übertragen. ... sie sind abstrakt einsetzbar - d.h. ohne allzu genaue Vorherbestimmung der Situationen, in denen sie konkret verwendet werden" (Luhmann 1981: 95). Genau dies zeigt jedoch ihre Ergänzungsbedürftigkeit, sobald es um situationsgerechte Anwendung geht.

Das gilt in besonderem Maße für die aus steuerungstheoretischer Sicht notwendige Gewährleistung der Handlungsbeurteilung. Im Kontext primär hierarchischer Steuerung, wie sie nach vorherrschender Auffassung für staatliches Handeln charakteristisch ist (bzw. sein soll), gewährleisten Recht und Geld als Vermittler von Entscheidungsprämissen grundsätzlich keine Prozesse der Handlungsbeurteilung oder Rückkoppelung, die herrschende Auffassung vertraut also lediglich auf "die Bindungswirkung von Entscheidungen". Prozesse der Handlungsbeurteilung müssen gesondert institutionalisiert werden.

Betrachtet man nun die Wirklichkeit der Verwaltungskontrolle, so stellt man fest, daß diese überwiegend in fragmentierter, dimensionsspezifischer Weise verläuft. Rechtmäßigkeit ist nur ein Kriterium des Verwaltungshandelns, dem man als weitere generelle Kriterien Zweckmäßigkeit, Wirtschaftlichkeit und für den Bereich der Daseinsvorsorge und der Leistungsverwaltung - Bürgernähe zur Seite stellen kann (vgl. Kaufmann (Hg.), 1979: bes. 18 ff., 505 ff.). Während die Beurteilung der Rechtmäßigkeit (durch die Gerichte) und der Wirtschaftlichkeit (durch die Rechnungshöfe) gesonderten staatlichen Instanzen übertragen ist, ist die Berücksichtigung der Kriterien der Zweckmäßigkeit und der Bürgernähe allenfalls in politisch-parlamentarischen Kontrollprozessen, sonst jedoch überwiegend nur durch verwaltungsinterne Kontrollen zu gewährleisten. Dabei scheint es sehr fragwürdig, inwieweit diese Kontrollen geeignet sind, eine Kommunikation über Ergebnisbewertung als Voraussetzung des Lernens der Gesetzes- und Verwaltungsevaluation, verstärkte Buher zusätzliche Formen der Einbezug von Experten und Interessenvertretere Bürgerbeteiligung, verstärkter

des Verwaltungshandelns gefordert.

Aus einer primär rechtsstaatlichen Perspektive erscheint diese Betonung der
rechtsexternen Dimensionen des Verwaltungs mindesten dann, wenn diese Kriterien Handlungandelns problematisch, zum geltenden Rechtsnormen diese Kriterien Handlungen nahelegen, die mit den ist jedoch gerade eine gewiscelieren. Aus steuerungstheoretischer Perspektive 
se selektiven verwaltungsexternen Kontrolle erforderlich, um der Multivalenz staatlicher Interventionen Rechnung zu tragen (vgl. Wirth 1986a). Öffentliche Verwaltungen stehen - wie übrigens alle gesellschaftlichen Akteure - typischerweise unter widersprüchlichen Anforderungen (hierzu grundlegend Hegner 1978) und sie vermögen daher die an sie gestellten Anforderungen nie vollständig, sondern bestenfalls in einer Form balancierender Optimierung zu erfüllen. Von daher muß auch das Problem der Verwaltungskontrolle neu bedacht werden (hierzu Hood 1986b).

Üblicherweise werden die Steuerungsmöglichkeiten durch Recht lediglich im Kontext der "Steuerung mittels regulativer Politik, dem Königsweg staatlicher Intervention" (Mayntz 1979: 55) diskutiert. In dieser Prioritätensetzung schwingt die typisch deutsche, hoheitliche Staatsauffassung mit, welche das 'Regierbarkeitsproblem' als Problem der Durchsetzbarkeit des gesetzlich formulierten staatlichen Willens interpretiert. Diese Auffassung impliziert streng genommen die notwendige Zweckmäßigkeit staatlicher Anordnungen, eine Art weiser Voraussicht des Gesetzebers über die zu erwartenden Folgen seiner Gesetze. Oder zum mindesten eine überaus hohe Lernfähigkeit und Problemverarbeitungskapazität des Gesetzgebers, der die Folgen der von ihm erlassenen Gesetze überprüft und sie entsprechend den gemachten Erfahrungen modifiziert. Endlich kann man diese Auffassung auch dahingehend deuten, daß sich der Gesetzgeber nur solcher Aufgaben annehmen solle, die mit den einfachen Mitteln regulativer Normierung ${ }^{24)}$ in zweckmäßiger und einigermaßen überschaubarer Weise gelöst werden können. Dieser liberal-konservativen Politikauffassung steht die faktische Entwicklung und der zum mindesten relative Erfolg wohlfahrtsstaatlicher Politik entgegen, deren Erfolgsbedingungen jedoch noch kaum in verallgemeinernder Form begreifbar sind.

Greifbares Ergebnis der gegenwärtigen Diskussion um 'Entrechtlichung' (hierzu bes. Voigt 1983b) ist denn auch weniger die Empfehlung totaler staatlicher Abstinenz als die des beschränkteren und indirekteren Einsatzes regulativer Normen als ultima ratio in einem Feld öffentlich-privater Interdependenzen. Die Empfehlungen gehen hier von 'Gesetzesentrümpelung' als bloßem Abbau überflüssiger oder gar widersprüchlicher Normen über die Flexibilisierung der Gesetzgebung durch vermehrten Einsatz von Generalklauseln und Ermessensspielräumen, die Verstärkung informellen Verwaltungshandelns (hierzu Bohne 1980) und vorgerichtlicher Konfliktregelung als Versuch gütlicher Einigung 'im Schatten des Rechts', bis zur "dezentralen Kontextsteuerung" korporatistischer Handlungssysteme.

Damit wird deutlich - was auch durch die einleitende Aufzählung unterschiedlicher Typen rechtlicher Normierung suggeriert werden sollte - daß "Steuerung durch Recht" einen weit umfangreicheren Bereich staatlicher Maßnahmen betriff, als dies in den vorherrschenden Diskussionen thematisiert wird. Gleichzeitig zeigt der Katalog der 'Entrechtlichungsvorschläge', daß sie 
nahezu alle in Abschnitt 2.1 angesprochenen Dimensionen der 'Verrechtlichung' betreffen, wobei jedoch keineswegs die kumulative Anwendung der verschiedenen Strategien empfohlen wird. Vielmehr zeichnen sich die 'Entrechtlichungsvorschläge' typischerweise dadurch aus, daß sie dysfunktionalen Wirkungen einer allzu weit getriebenen Verrechtlichung durch Abschwächung oder Beseitigung von einzelnen Aspekten begegnen wollen. Für die Beurteilung der wohlmeinenden Vorschläge fehlt es jedoch bisher weitgehend an klaren Kriterien, was bei der Unklarheit des zugrunde liegenden Steuerungsbegriffs nicht überrascht.

Wenn wir soziale Steuerung allgemein als das Problem von Handlungskoordination fassen, also der Ermöglichung erwartbarer Anschlußhandlungen im Horizont eines übergreifenden Kontextes, so werden die meisten 'Entrechtlichungsvorschläge' plausibel: Gesetzesentrümpelung meint die Beseitigung von Vorschriften, die entweder keine oder 'falsche' Anschlußhandlungen wahrscheinlich machen; die Flexibilisierung von Gesetzgebung und Verwaltung soll ein verstärktes Eingehen auf die Besonderheiten des in Frage stehenden Einzelfalles ermöglichen und auf diese Weise 'einsichtige' Anschlußhandlungen von Verwaltung und Publikum erleichtern; Informalisierung der Konfliktregelung in Verwaltung und Justiz soll die Orientierung an Rechtsnormen in der Interaktionssituation zurückdrängen und auf diese Weise sachlich gerechtfertigte Kompromisse erleichtern; und die unter dem Stichwort 'Neokorporatismus' diskutierten Verhandlungssysteme zwischen Staat und Verbänden sollen der 'Interessenvermittlung' zwischen Akteuren dienen, die aufgrund ihrer unterschiedlichen Struktur und Handlungsmöglichkeiten im Falle eines antagonistischen Verhaltens mehr zu verlieren als zu gewinnen hätten.

\subsection{Recht, Organisation und Person}

Was nun die Wirkungsmöglichkeiten der Gesetzgebung betrifft, so hängt sie entscheidend von der angemessenen Einschätzung der sozialen Folgen bestimmter Normen ab. "Rechtsänderungen als Einzelmaßnahmen (werden) in mehr oder weniger unifunktionaler, zweckgerichteter Perspektive entschieden, (treffen) aber auf eine durchweg multifunktionale Wirklichkeit auf... Diese Multifunktionalität gibt jeder einzelnen Neuerung eine hohe, zumeist unermeßliche Zahl von Folgewirkungen in ganz andersartigen Sachbereichen" (Luhmann 1972: Rechtsgebrauchs es durchaus als plausibel erscheinen läßt, daß die Rechtswirkungen im durchschnittlichen Fall so unberechenbar nicht sind. Welches aber scheinlich oder unwen, welche die Absehbarkeit von Rechtswirkungen wahr- 
hier genannt, die jedoch je nach Typus des Normadressaten - Organisationen oder Personen - unterschiedliche Bedeutung besitzen:

1. Die Kompatibilität oder Inkompatibilität von Rechtsnormen mit im zu regelnden Kontext anerkannten Sozialnormen oder Werten, ein in der Rechtswissenschaft seit langem erkannter und diskutierter Zusammenhang;

2. die Kompatibilität oder Inkompatibilität von Rechtsnormen mit Interessenlagen der Normadressaten, ein vor allem in der Politikwissenschaft hervorgehobener Zusammenhang;

3. die Etabliertheit und Zugänglichkeit des Handlungszusammenhangs, in den rechtliche Regulierung eingreift, ein vorwiegend soziologischer Gesichtspunkt. Unter Etabliertheit sei der Umstand verstanden, daß bestimmte Handlungszusammenhänge bereits solange existieren, daß die in ihnen Handelnden eigene Situationsdeutungen aufgebaut und Handlungsroutinen entwickelt haben, daß also die Verhaltensweisen im betreffenden Kontext zum mindesten unter den Beteiligten im Regelfall erwartbar sind. Wo es an derartigen sozialen Regelmäßigkeiten überhaupt fehlt, kann naturgemäß auch die Auswirkung von Interventionen nicht vorausgesehen werden. Die sozialen Regelmäßigkeiten müssen jedoch überdies für Dritte, insbesondere solche, die mit Politikformulierung befaßt sind, bekanntwerden, und dies setzt im Regelfall eine zum mindesten beschränkte Öffentlichkeit oder selbst rechtlich gesicherte Zugänglichkeit voraus. ${ }^{26}$

Bei der Einschätzung möglicher Auswirkungen rechtlicher Regulierungen muß auf einen in der Rechtstheorie und Gesetzgebungslehre bisher nicht systematisch berücksichtigten Unterschied hingewiesen werden, die Frage nämlich, ob es sich bei den Normadressaten um Organisationen oder natürliche Personen handelt. ${ }^{27)}$ Im Kern läßt sich die Bedeutung dieses Unterschieds auf die Affinität von Recht und Organisation einerseits und die Fremdheit von Recht und interpersoneller Kommunikation andererseits zurückführen. Unabhängig vom inhaltlichen Verhältnis zwischen spezifischen Rechts- und Sozialnormen kann für formal organisierte Handlungskontexte eine verrechtlichte Kommunikationsform als normal gelten, während sie für Interaktionen, die auf der Einschätzung wechselseitiger Vertrauenswürdigkeit der Interaktionspartner beruhen, als Vertrauensbruch in Erscheinung treten kann (vgl. Ellscheid 1979: $42 \mathrm{ff}$.). Vor allem für Personen mit geringer Organisationserfahrung - etwa Arbeiter, Jugendliche oder Hausfrauen - kann die an Recht orientierte Kommunikationsform, wie sie insbesondere für Verwaltungskontakte charakteristisch ist, schon von sich aus einschüchternd, um nicht zu sagen abstoßend wirken. ${ }^{28}$ Allerdings ist wohl auch hier noch näher zu unterscheiden: Es gibt Rechtsmaterien, deren normativer Gehalt aufgrund ihrer lange dauernden Geltung (ihrer 'Etabliertheit') auch ohne genaue Rechtskenntnis in großen Zügen Bestandteil des Rechtsbewußtseins weiter Bevölkerungskreise geworden ist, so 
insbesondere im Privat- und Strafrecht. Öffentlichrechtliche Verhältnisse, insbesondere die Materien des Verwaltungsrechts, bleiben dagegen dem Rechtsbewußtsein der Bevölkerung fremd. Überdies irritieren Rechtsänderungen das vorhandene Rechtsbewußtsein, und insoweit die Änderbarkeit des Rechts - also gerade der unter Steuerungsgesichtspunkten immanente Vorteil der Positivierung - ins öffentliche BewuBtsein tritt, ist mit einem Unbestimmtwerden des Rechts- und Unrechtsbewußtseins sowie einer Irrationalisierung des Rechtsgefühls zu rechnen (vgl. Kaufmann 1985a). Man wird also auf der Ebene natürlicher Personen - soweit sie nicht als Spezialisten alltäglichen Kontakt zu bestimmten Rechtsmaterien besitzen - immer weniger ausreichende Rechtskenntnis voraussetzen dürfen. Inwieweit diese durch ein generelles Vertrauen in die Wirksamkeit der Rechtsordnung und die daraus folgende Bereitschaft, sich in einschlägigen Fällen an kompetente Spezialisten der Rechtspflege oder der Verwaltung zu richten, substituiert werden kann, ist eine noch weitgehend
unerforschte Frage. ${ }^{29}$.

Ganz anders dagegen sind die Verhältnisse im Bereich formaler Organisation: Je größer und differenzierter eine Organisation ist, desto mehr dominiert schriftliche über mündliche Kommunikation und desto selbstverständlicher wird die Substituierbarkeit der Interaktionspartner. Überdies sind - zum mindesten in hierarchisch strukturierten Organisationen - dem regulativen Recht analoge Normierungen auch außerhalb des öffentlichen Bereichs verbreitet. Der gesamte Interaktionsstil ist hier also für rechtsförmige Regelungen weit offener, und man wird von einer hohen formalen Akzeptanzbereitschaft rechtlicher Normierung ausgehen können. Dies ist insbesondere von Bedeutung für den Bereich der formal organisierten Akteuren wie - also etwa zwischen staatlichen und anderen modernen Kliniken. Zumeist verfügen diese Organisationen auchehmungen oder spezielle Rechtsabteilungen oder zum mindesten über spezialisierte Fachkräfte, die sich in den für sie relevanten Rechtsmaterien auskennen.

Aus dieser grundsätzlich hohen Affinität zur rechtlichen Regelung resultiert auf seiten organisierter Akteure allerdings auch eine Tendenz zum instrumentellen Rechtsgebrauch, mit der bei natürlichen Personen nur in Ausnahmefällen gerechnet werden muß. Hierunter sei die Instrumentalisierung von Rechtsnorlichen Regulierung zuwiderläuft. Häufiger wenn dies den Zwecken der rechtdaher in diesem Bereich der Versuch, die als offene Rechtsverletzungen ist Politik zu umgehen, indem Gesetzeslücken intendierten Wirkungen regulativer auch konkurrierende Rechtsnormen zur Legitimation des eigenen Handelns Instanzen wie organisierter Normadressaten - und zwar sowohl auf seiten staatlicher Prinzip erhoben, worauf sich ein dauernder - die Positivität des Rechts zum normen zu ergeben scheint. Es uaernder Bedarf an Modifikation der Rechts- 
Aufstellung von Hasskarl (1968, zit. nach Noll 1973: 165) zwischen 1949 und 1965 fast die Hälfte aller Bundesgesetze und über die Hälfte der Verordnungsermächtigungen die Gebiete des Finanzwesens und des Wirtschaftsrechts betreffen. "Diese Rechtsbereiche sind also unstabiler und enthalten weniger auf Dauer angelegte Normen als die übrigen" (Noll). Die wohlfahrtsstaatlichen Kernmaterien des Arbeits- und Sozialrechts, die häufig als Hauptursache der 'Gesetzesflut' vermutet werden, treten mit $14,3 \%$ der Gesetze - zum mindesten in der untersuchten Epoche - deutlich zurück. ${ }^{30}$

\section{Die Bedeutung des Rechts im Kontext unterschiedlicher Formen sozialstaat- licher Intervention}

Es verbleibt nun, die bisherigen Überlegungen im Hinblick auf die Steuerbarkeit sozialstaatlicher Interventionen durch Recht zusammenzufassen.

Hierbei ist zunächst die Mehrstufigkeit dieser Interventionen selbst hervorzuheben, die für den Fall sozialer Dienstleistungen durch Figur 1 schematisch veranschaulicht wird.

Diese Darstellungsweise hebt auf die verschiedenen Voraussetzungen ab, von denen die Erbringung sozialer Dienstleistungen abhängig ist, führt jedoch nicht die beteiligten Akteure auf und verweist auf unterschiedliche Politik- und Verwaltungsebenen nur durch die Unterscheidung von örtlichem und überörtlichem Bereich. ${ }^{31)}$ Immerhin wird die Distanz zwischen dem nur an Phase A beteiligten Gesetzgeber und den nur an Phase $D$ beteiligten Leistungsempfängern oder Zielgruppen deutlich. Die Phasen A bis $\mathrm{C}$ vollziehen sich im wesentlichen auf der Basis von Inter-Organisationsbeziehungen, wobei in Phase A staatliche Akteure (Parlament, Ministerialverwaltung) dominieren, allerdings unter mehr oder weniger tatkräftiger Mitwirkung von Parteien und Verbänden. In Phase B dominieren administrative Akteure auf kommunaler, Landes- und Bundesebene, die - über Haushaltsvorbereitung und Haushaltsabwicklung die öffentliche Teil- oder Totalfinanzierung sozialer Leistungen bestimmen, unter meist tatkräftiger Mitwirkung der interessierten Träger von Leistungsangeboten. Letztere - teils staatliche, teils kommunale, teils 'freie Träger' dominieren in Phase $\mathrm{C}$, indem sie etwa über Errichtung, Ausbau oder SchlieBung von Schulen, Krankenhäusern, Kindergärten oder Beratungsstellen beschließen, stets allerdings unter einer mehr oder weniger starken staatlichen Aufsicht, in den Flächenstaaten meist durch Mittelinstanzen (Bezirksregierung, Schulkollegien). Phase D endlich wird typischerweise vom leistungserbringenden Personal bzw. dessen unmittelbaren Vorgesetzten dominiert, bei beschränkten Mitwirkungsmöglichkeiten der Leistungsadressaten einerseits und der Einrichtungsträger andererseits. 


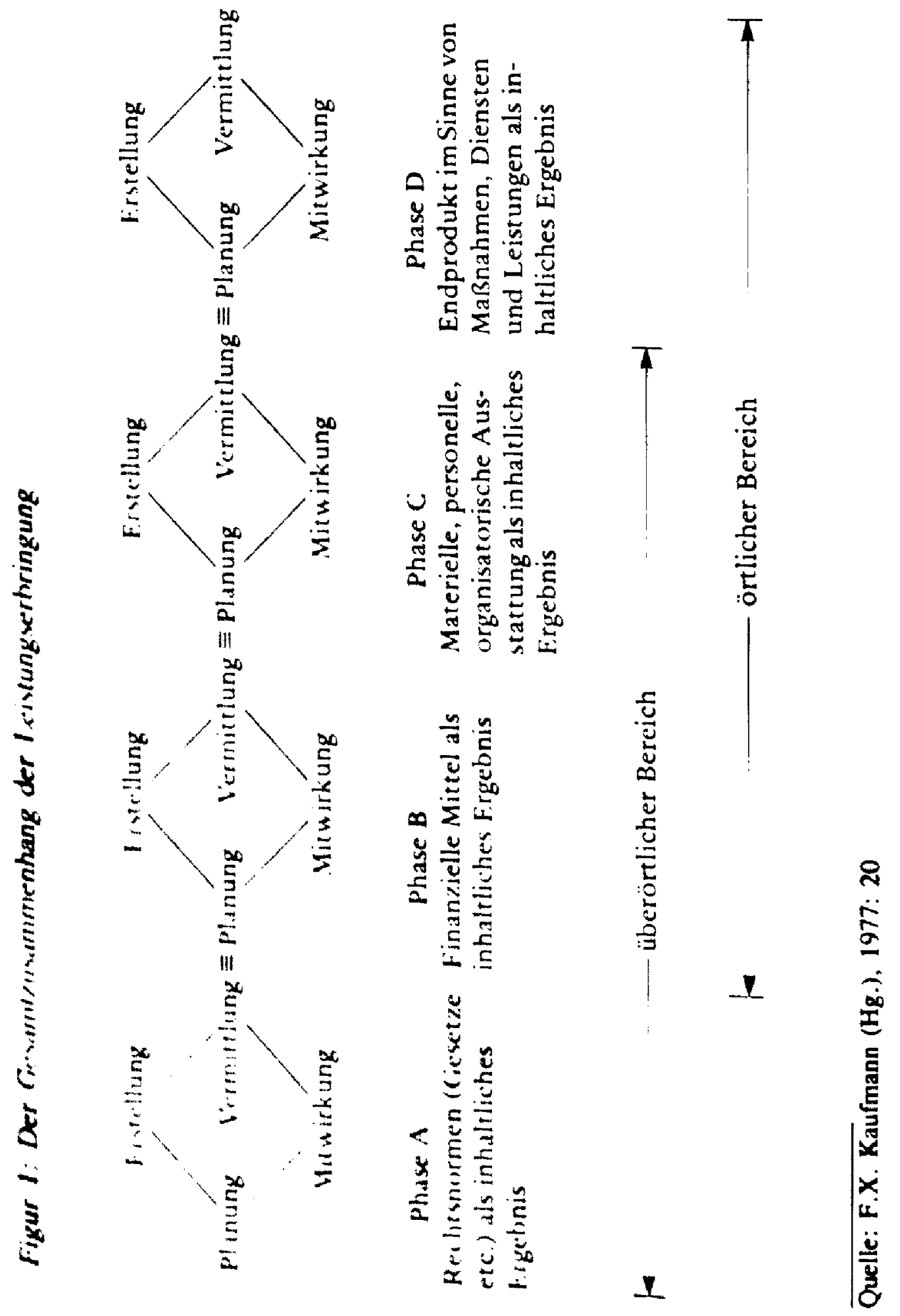




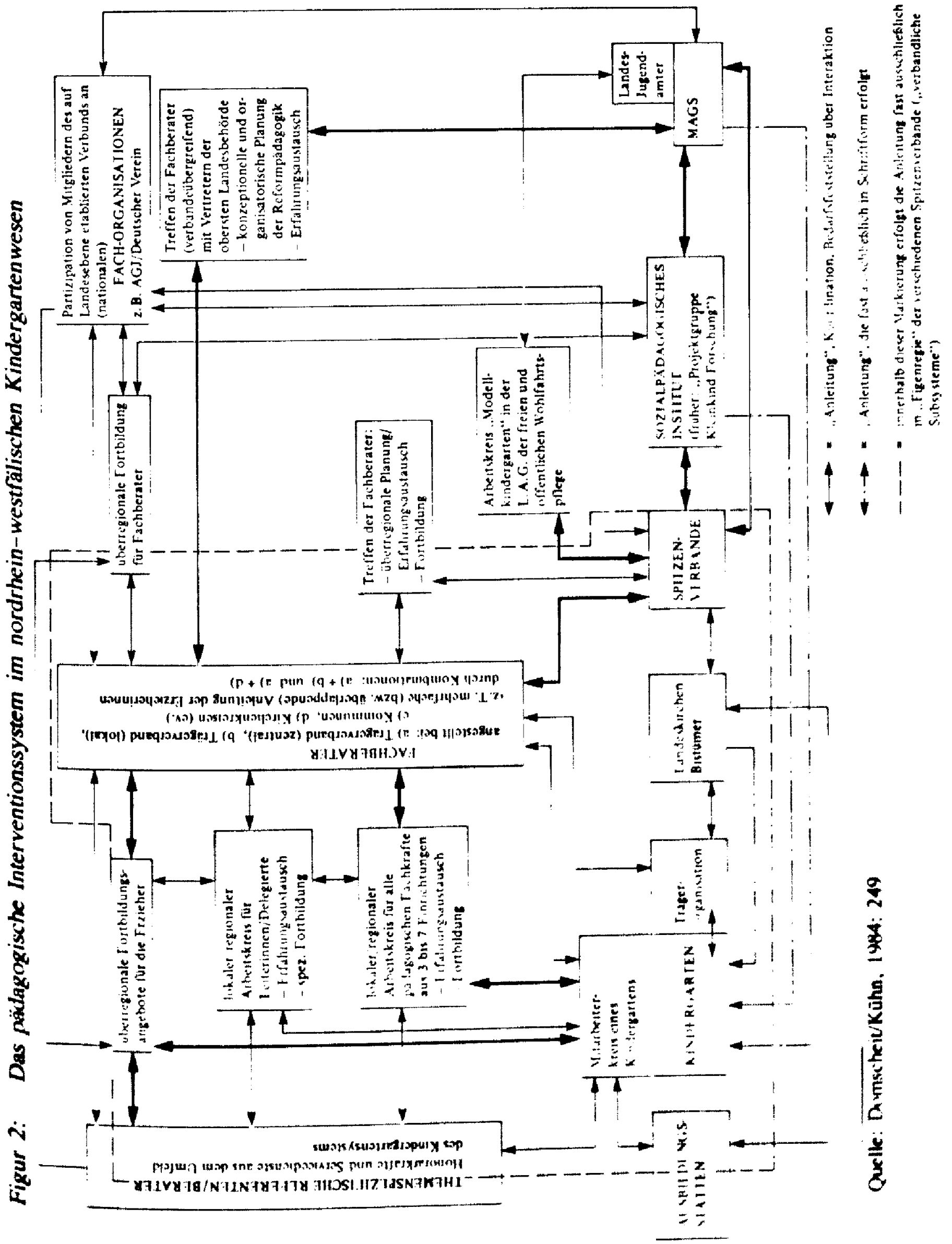


Einflußmöglichkeiten der auf einer bestimmten Stufe dominierenden Akteure auf Handlungsverläufe anderer Stufen sind beschränkt, wobei höhere Instanzen immerhin die Entscheidungen nachgeordneter Instanzen durch Bedingungen beeinflussen können, während Einflüsse 'von unten' nur in Ausnahmefällen und auf meist indirektem Wege (z.B. über die Presse oder die Gerichte) zum Tragen kommen können. Für andere Bereiche wohlfahrtsstaatlicher Intervention müßte die Darstellung etwas modifiziert werden, doch würde sich am grundlegenden Sachverhalt nichts ändern, daß nämlich die Normbenefiziare als natürliche Personen völlig an der Peripherie des häufig imposanten Netzwerks interorganisatorischer Beziehungen stehen, innerhalb dessen die staatliche Intervention sich verwirklicht. Ein immer noch vereinfachtes Beispiel eines konkreten Netzwerks, wie es im Rahmen einer Studie zur Implementation des Nordrhein-Westfälischen Kindergartengesetzes rekonstruiert wurde, zeigt

Um die spezifischen Steuerungsmöglichkeiten des Rechts und ihre Grenzen im Bereich der Sozialpolitik deutlicher herauszuarbeiten, empfiehlt es sich, nicht nach institutionellen Gebieten der Sozialpolitik, wie sie durch die Systematik der Gesetze und Träger präformiert ist, sondern nach dem Typus der Intervention zu unterscheiden. Dabei erscheint unter wohlfahrtstheoretischen Gesichtspunkten eine Klassifikation angezeigt, die die angestrebten Wirkungen mit den hierfür typischerweise geeigneten Instrumenten verbindet. ${ }^{32)}$ Ausgangspunkt ist die Frage nach den Voraussetzungen sozialer Teilhabe oder - gesellschaftstheoretisch formuliert - Inklusion. ${ }^{33)}$ Soziale Teilhabe oder die Realisierbarkeit grundrechtlicher Freiheiten als Inbegriff wohlfahrtsstaatlicher Zielsetzung setzt nach der hier vorgeschlagenen Schematisierung viererlei voraus (vgl. Figur 3):

1. Status, d.h. sozial anerkannte Teilhaberechte, wie sie in den verfassungsmäßigen Grundrechten oder gesetzlichen Ansprüchen präformiert sind. Ihre soziale Verwirklichung setzt allerdings deren faktische Respektierung durch
Dritte in Prozessen der Statuszuweisung voraus.

2. Ressourcen: Hierunter seien knappe Güter (bes. Zeit, Geld, Besitz) verstanden, über die Individuen grundsätzlich frei, jedoch in beschränktem Umfange verfügen können. Alle Formen sozialer Teilhabe verbrauchen Ressourcen, die somit je nach Umständen zum Engpaßfaktor werden

3. Gelegenheiten: Soziale Teilhabe setzt ein Angebot an Teilhabemöglichkeiten voraus, das nach Maßgabe von Status, Ressourcen und Kompetenzen wahrgenommen werden kann. Im Unterschied zu Ressourcen handelt es sich hier um Aspekte der sozialen, räumlichen und sachlichen Umwelt der Individuen, über die diese nicht selbst, sondern nur nach von Dritten Bedürftigkeit oder Kooperationsbereitschaft) verfügen können. 


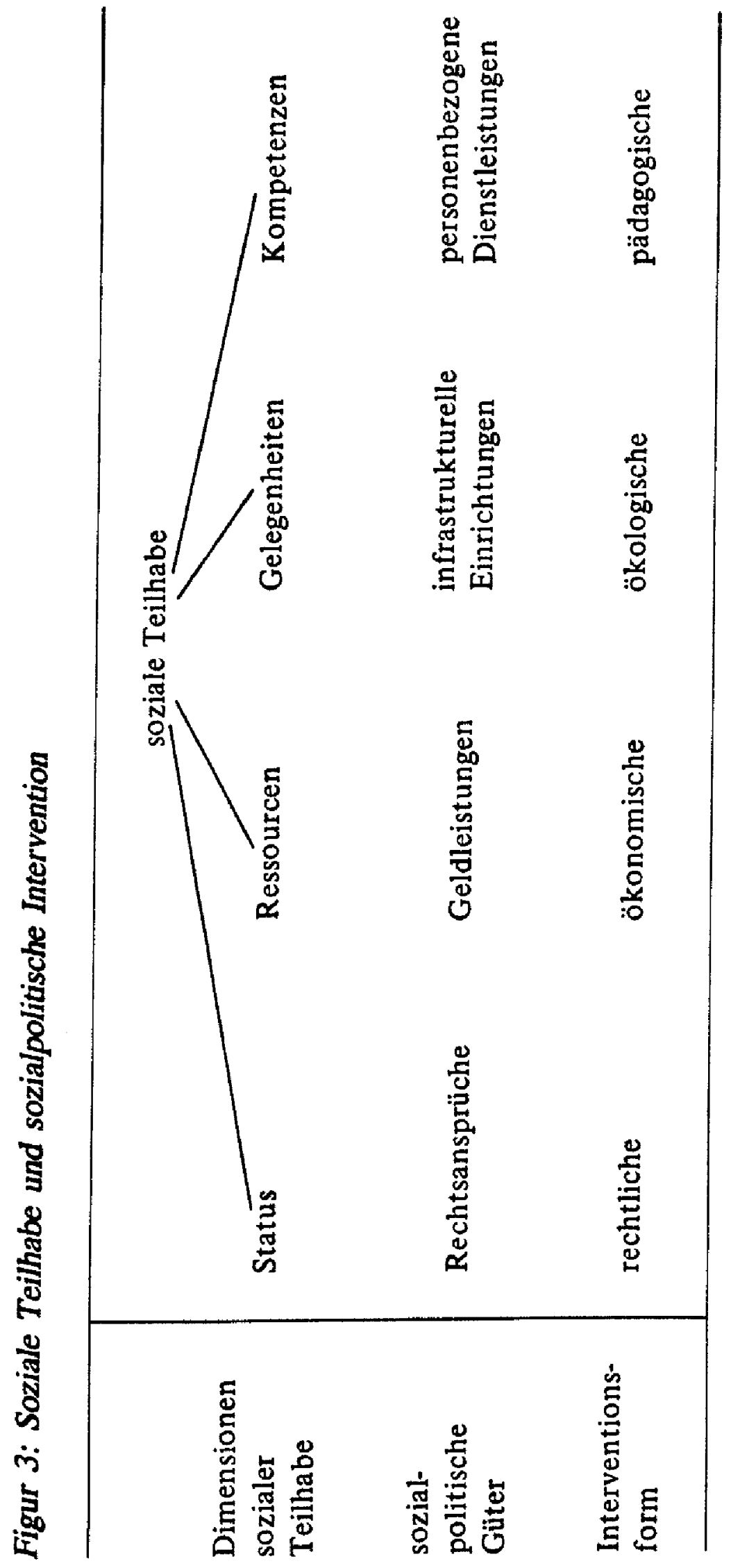


4. Kompetenzen: Hierunter werden unmittelbar a die eigene Person gebundene Voraussetzungen der Handlungsfahigkeit (wie Bildung, Gesundheit, Wissen, Fertigkeiten) und der Handlungsbereitschnft (wie normative Orientierungen, Leistungsbereitschaft oder spezifische Motivationen) verstanden, die als notwendige Bedingungen wozialer Attivitu getten können.

Sozialstaatliche Interventionen kōnnen im Hinblick auf die mit ihnen verbundenen Intentionen danach klassifiziert werden, welche dieser Dimensionen der Prämissen sozialer Teilhabe sie zu fördern geeignet sind. ${ }^{30)}$

Auch die 'sozialpolitischen Güter' lassen sich in Anknüpfung an verbreitete Klassifikationen in vier Kategorien - Rechtsansprüche, Geldleistungen, infrastrukturelle Einrichtungen und personenbezogene Dienstleistungen einteilen, die sich den vier Dimensionen sozialer Teilhabe in meist eindeutiger Weise zuordnen lassen. Dementsprechend können sozialstaatliche Maßnahmen, deren Endzweck in der Herstellung oder Vermitulung dieser vier Leistungstypen zu sehen ist, unter den Begriff der zugeordneten (rechtlichen, ökonomischen, ökologischen und pädagogischen) Interventionsform subsumiert werden. Die nebenstehende Übersicht konkretisiert das Konzept in einigen wesentlichen
Punkten.

Bezogen auf diese Interventionsformen sei nun abschließend die Bedeutung rechtlicher Normierung spezifiziert. ${ }^{35)}$

\subsection{Rechtliche Interventionsform}

Wie schon der Name sagt, kommt hier dem Recht und den damit verbundeÜ̈nerwiegend der Rechtsaufsicht und der Rechtspflege zentrale Bedeutung zu. Setzung und Anwelt es sich bei der rechtlichen Interventionsform um die schwert die Wirksamkeit - Normadressaten, wobei jedoch - und dies eridentisch sind. So ist etwa der Arbeitgeber gehalten, im Interesse der Gesund heit des Arbeitnehmers bestimmte Vorkehrungen zu treffen, zu deren kompetenter Ausgestaltung Werksärzte oder Sicherheitsingenieure zuständig sind, während die Überwachung bei staatlichen Gewerbeärzten oder Gewerbeaufsichtsämtern liegt. Dem Arbeitnehmer steht kein unmittelbarer ErfüllungsSozialrecht - dem im Falle der Schädigung. Aber auch dort, wo - wie im also selbst auch Normadrenefiziar subjektive Rechte eingeräumt werden, er kenntnis und mangelnde Kompeten verhindert dann oft subjektive Rechtsuntionskonforme Rechtswahrnehmung. Im Umgang mit Behörden eine inten- 


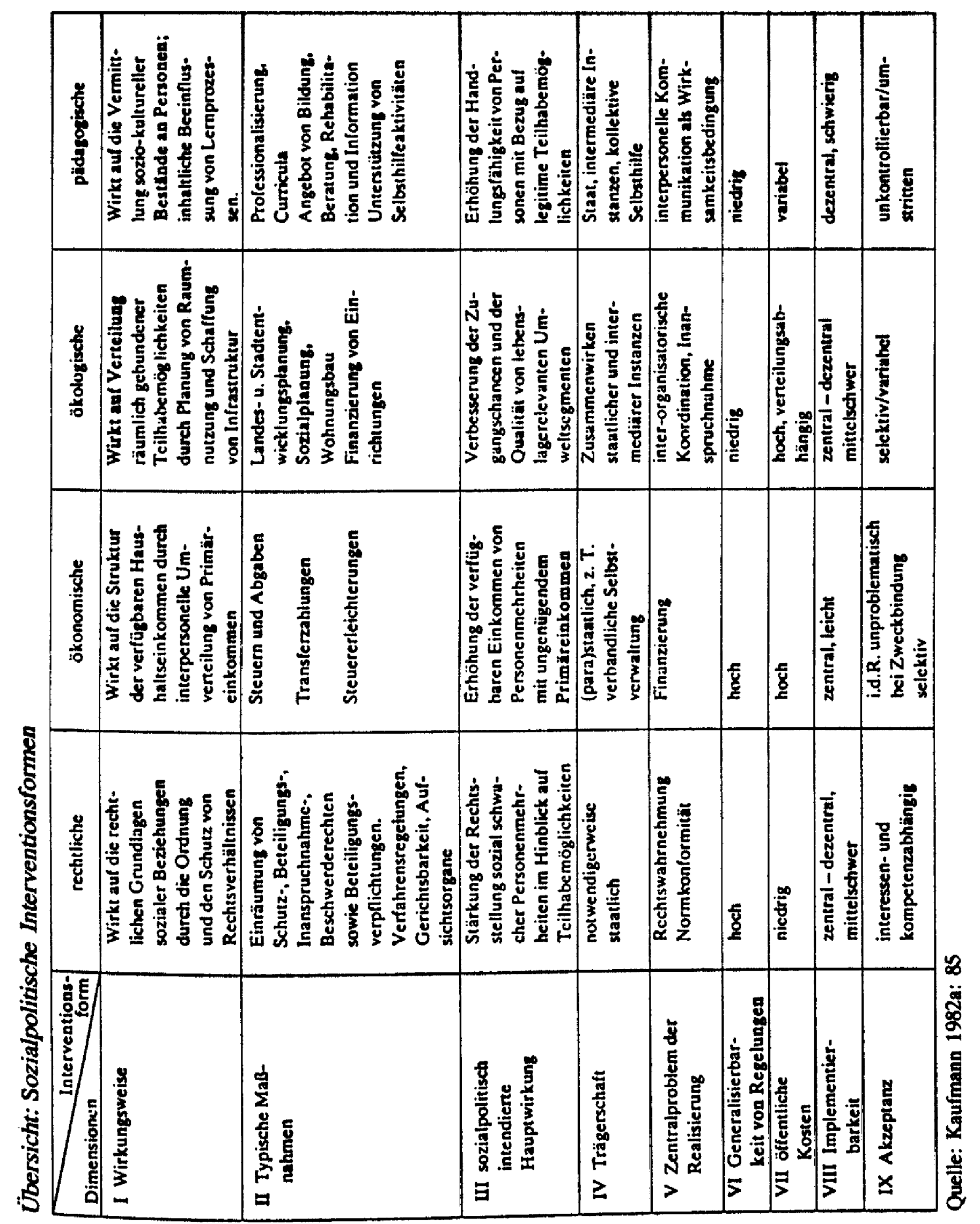


Selbsthilfe (z.B. nachbarschaftlicher, solidarisch-betroffener oder gewerkschaftlicher Art) greifen, kann u.U. durch pädagogische Interventionen (z.B. Sozialarbeit) flankierend geholfen werden .

Vor allem im Bereich des Arbeitsrechts muß überdies damit gerechnet werden, daß die Rechtsansprüche und Schutznormen der Arbeitnehmer Interessen der Arbeitgeber verletzen und von daher Implementationswiderstände zu erwarten sind. Es hängt dann von der Präsenz oder Mobilisierbarkeit staatlicher Aufsichtsbehörden $a b$, ob sich der Umfang der Normverletzungen ausdehnt. ${ }^{36)}$ Alles in allem kann jedoch davon ausgegangen werden, daß in diesem auf die Wirkungsmöglichkeiten des Rechts ja spezifisch zugeschnittenen Bereiche Steuerungswirkungen durch Recht vergleichsweise einfach zu erzielen sind. Insoweit als die Implementation der Rechtsnormen allerdings einen spezifischen Kontrollaufwand nach sich zieht, ergeben sich auch hier jeweils spezifische Wirkungsbedingungen, die bereits im Gesetzgebungsprozeß mit berücksichtigt
werden sollten.

\section{2. Ökonomische Interventionsform}

Auch die Administration des flexiblen Interventionsmediums Geld läßt sich grundsätzlich durch Rechtsnormen vergleichsweise leicht steuern - der entscheidende Engpass ist hier die Verfügbarkeit der Mittel selbst. Allerdings ist zu unterscheiden zwischen Geldleistungen, die aufgrund allgemeiner und einfach nachweisbarer Titel der Anspruchsberechtigung zugesprochen werden (wie z.B. Altersrenten oder Kindergeld) und solchen, wo die Anspruchsberechtigung nur aufgrund von Bedingungen entsteht, deren Vorliegen im Einzelfall geprüft werden muß (wie z.B. bei der Invalidenrente, dem Wohngeld oder der Hilfe zum Lebensunterhalt). Im letztgenannten Fall sind die Mitwirkungserfordernisse der Normbenefiziare wesentlich höher, und damit auch deren Einflußmöglichkeit auf die Höhe der gewährten Leistung. Das kann - bezogen auf den Rechtszweck - sowohl zu Unterforderungen wie zu Überforderungen (hierzu Wulfhorst 1982) führen. Während die Administration von Geldleistungen, auf die ein wiederkehrend-gleichbleibender Anspruch besteht, leicht zentralisiert werden werden soll, eine derürche, deren Bestandsvoraussetzung stets neu festgestellt kommunale Ämter mit dentrale Administration existieren; häufig werden dann einer einheitlichen Recht Implementation betraut, wobei vielfältige Einflüsse

Staatliche $\mathrm{MaR}$.

sich in der Bundesrepun zur Erhöhung der verfügbaren Einkommen bedienen eingehenden rechtlichen grundsätzlich administrativer Mittel, die auf einer terungen als auch für die ing beruhen. Das gilt sowohl für SteuererleichTransferzahlungen, welche in dieser Betrachtung im Vordergrund stehenden 
subjektiver Rechtsansprüche gegenüber staatlichen oder parastaatlichen Sozialleistungsbehörden gewährt werden. ${ }^{37}$ Angesichts der vielseitigen Verwendungsmöglichkeiten des Geldes und der daraus resultierenden universalen Begehrtheit wird der Umgang mit Geld staatlicherseits regelmäßig sehr detailliert geregelt. Ökonomische Interventionen beruhen daher regelmäßig auf einer detaillierten konditionalen Programmierung in der Form von Gesetzen, Verordnungen und verwaltungsinternen Ausführungsbestimmungen. Auch im Rahmen der ökonomischen Interventionsform kommt also dem Recht - im Sinne der Einräumung subjektiver Rechtsansprüche, der Regelung von Verfahren und der Festsetzung von Bedingungen - große Bedeutung zu.

Voraussetzung zielgerechter Rechtswirkungen ist hier im Regelfall die Wahrnehmung der subjektiven Rechtsansprüche in der Form ordnungsgemäßer Antragstellung sowie eine korrekte Sachverhaltsermittlung seitens der zuständigen Sozialbehörden. Dabei ergibt sich ein charakteristisches Dilemma: Einerseits liegt die Realisierung eingeräumter Rechtsansprüche auch und gerade für die bedürftigsten Bevölkerungsgruppen (welche häufig auch die geringste Handlungskompetenz aufweisen) im Sinne des Rechtszwecks. Daraus ergibt sich die Forderung an die Sozialbehörden, den Normbenefiziaren bei der Realisierung ihrer Ansprüche behilflich zu sein. Andererseits sind sie jedoch auch gehalten, die Realisierung ungerechtfertigter Ansprüche zu verhindern und insbesondere Versuchen eines instrumentellen Rechtsgebrauchs entgegenzutreten. ${ }^{38)}$ Diese gleichzeitig helfende und kontrollierende Doppelrolle scheint im Publikumskontakt schwer realisierbar, was im Rahmen der Sozialämter zur Trennung von 'Innendienst' und 'Außendienst' geführt hat (vgl. Zacher 1984: $63 \mathrm{f}$.). Erschwert wird eine sachgerechte Rechtsanwendung weiterhin, wenn - wie heute im Bereich der Sozialhilfe - die Sachverhaltsfeststellungen im Horizont von Massenarbeitslosigkeit und restringierten Budgets erfolgen müssen (vgl. Leibfried/Tennstedt 1985).

Ähnlich wie die rechtliche setzt die ökonomische Interventionsform eine 'Verrechtlichung' im fünffachen oben beschriebenen Sinne zwingend voraus. Umverteilung der Primäreinkommen, und dies ist der funktionale Kern dieser Interventionsform, setzt auf der Seite des Nehmens hoheitliche Akte und auf der Seite des Gebens die Einhaltung eindeutig definierter Bedingungen voraus. Berücksichtigt man, daß nur über die Einkommensumverteilung von den Erwerbstätigen zu den Nicht-Erwerbstätigen das Verhältnis zwischen den Generationen ökonomisch stabilisiert werden kann (vgl. Kaufmann/Leisering 1984), wird die Zentralität dieser Interventionsform deutlich. 


\section{3 Ökologische Interventionsform}

Während 'Daseinsvorsorge' und die damit einhergehende Planung und Bereitstellung kollektiv oder individuell nutzbarer Einricktungen von alters her eine gemeindliche Aufgabe gewesen ist (vgl. Gröttrup 1973) hat sich hier vor allem aufgrund der daraus resultienenden regionalen und sozialen Disperit:ten - in den letzten Jahrzehnten ein wachsender Stanseinflub durchgesetz. Erinnert sei etwa an den sozialen Wohnungabau, des Studtebauforderungsgesetz oder das Krankenhausfinanzierungsgewetz, aber auch auf Lladerebene hat der Staatseinfluß in weiten Bereichen zugenommen. Ein besonderes Kapitel stellen in diesem Zusammenhang die sog. Gemeinachaftsaufgaben (Art. 91a und b GG) dar, bei denen neben der örtlich notwendigen Implementation ein Zusammenwirken von Bund und Ländem erfonderlich ist, das zu spezifischen Koordinationsschwierigkeiten geführt hat (vgl. Scharpf u.a. 1976).

Ziel sozialstaatlicher okologischer Intervention ist 'die Angleichung der Lebensbedingungen' in der Bundesrepublik Deutschland. Die spezifischen kumulativen Benachteiligungen, die sich aus der unterschiedlichen Wirtschartskraft bestimmter Regionen, den unterschiedlichen Praferenzen kommunaler Mehrheiten, der unterschiedlich entwickelten Nachfrage nach bestimmten Dienstleistungen (z.B. Bildung) usw. ergeben (vgl. hierzu Kaufmann 1979, passim), sollen durch staatliche Eingriffe reduziert werden. Dem dient teilweise die Verstaatlichung von Planungskompetenzen, vor allem aber ein vielfaltiges System der Finanzhilfen, durch die das Planungs- und Investitionsverhalten der Kommunen in Richtung auf eine Verbesserung der räumlichen und sozialen Infrastruktur beeinflußt werden soll. Auch wenn derartige Programme im Einzelfall durchaus auch konjunkturelle oder regionalpolitische Motive haben mögen, so interessiert aus sozialpolitischer Sicht ihre Wirkung auf die Lebenslage der im Einzugsgebiet bestimmter Einrichtungen wohnenden Bevölkerungs-
gruppen. Erweiterung der Teilhabechancen dar.

Dennoch stellt die Feststellun dar.

von Prioritäten nach derartigen des Bedarfs und insbesondere die Abwägung weitgehend ungelöstes Problem Einrichtungen ein in systematischer Form unterscheiden: Problem dar. Verschiedene Positionen lassen sich

a) Anhänger einer möglichst weitgehenden 'Privatisierung' oder 'Vermarktlichung' der Güterproduktion argumentieren, der vorhandene Bedarf lasse
sich komparativ Einrichtungen zu Markt- oder zum mindesten die Leistungen dieser (öffentlich oder privat) angeboten werden weitgehende Konkurrenz zwischen verden. Dabei soll eine möglichst Gegen diese Position ist zunăchst kritisch 
le Angebote, welche typischerweise entweder in der Form von 'Selbstbedienung' (z.B. Sportstätten, Gemeindehäuser) oder in der Form der Inanspruchnahme sozialer Dienstleistungen (z.B. Schulen, Krankenhäuser) genutzt werden, eine der wesentlichen Eigenschaften 'reiner' Wirtschaftsgüter, nämlich ihre Mobilităt, entbehren. Infrastrukturelle Einrichtungen sind per definitionem ortsgebunden, d.h. ihre Inanspruchnahme setzt die Mobilitä der Nachfrager und nicht der Anbieter voraus. Gerade für sozial benachteiligte Gruppen ist jedoch eine unterdurchschnittliche Mobilität anzunehmen; manche Einrichtungen (z.B. Kindergärten) sind für bestimmte Bevölkerungsgruppen nur zugänglich, wenn sie in Fußgängernähe angeboten werden. Eine angemessene räumliche Streuung des Angebots ist jedoch bei rein marktmäßiger Steuerung nicht $\mathrm{zu}$ erwarten. Zum mindesten müßten hier staatlich oder gemeindlich koordinierte Abstimmungsprozesse zwischen den Anbietern stattinden, wie sie etwa im Bereich der Jugendhilfe oder der Krankenhausfinanzierung versucht werden.

b) Typischerweise werden infrastrukturelle Einrichtungen jedoch nicht nur öffentlich koordiniert, sondern auch in ihrer Inanspruchnahme öffentlich subventioniert. Leistungen des Bildungssystems sind in der Bundesrepublik weitgehend unentgeltlich, die Inanspruchnahme von Krankenhäusern deckt nur die variablen Kosten, die Nutzung von Kindergärten wird von Eltern, Trägern und Staat gemeinsam finanziert usw. Der rationale Gehalt dieses 'Ökonomisch irrationalen' Vorgehens liegt in der Unterstellung, daß die Nutzung derartiger Einrichtungen nicht nur einen individuellen, sondern auch einen kollektiven Nutzen stiftet, daß die Inanspruchnahme dieser Einrichtungen also 'meritorious' sei, daher der ökonomische Begriff der meritorischen Güter. Es wird also ein öffentliches Interesse an der Inanspruchnahme dieser Güter unterstellt, auch dann, wenn die Nutzeneinschätzung der potentiellen 'Konsumenten' nicht so hoch ist, daß sie bereit wären, die vollen Produktionskosten zu bezahlen. ${ }^{39)}$ Die Inanspruchnahme infrastruktureller Einrichtungen dient - so die meist nur implizite Begründung - der Entfaltung von Humanvermōgen, d.h. von Kompetenzen, die gesellschaftlich erforderlich sind: Arbeitskraft, Erziehungsfahigkeiten, politische Kompetenz usw. (hierzu grundsätzlich Krüsselberg 1977). Es ist also offensichtlich, daß gerade in diesem Bereich über wesentliche Bedingungen der Gewährleistung einer sozialen Verwirklichung der verfassungsmäfigen Grundrechte entschieden wird.

c) Von diesen grundsätzlichen sozialstaatlichen Begründungen her läßt sich allerdings eine Bedarfsnormierung noch nicht ableiten. Eine Obergrenze ist dort zu vermuten, wo das Angebot auch bei unentgeltlicher Nutzungsmöglichkeit nicht mehr voll in Anspruch genommen wird, wobei auch hier wie das erfolgreiche Beispiel der Bildungswerbung auf dem Lande zeigt, die Grenzen hinausschiebbar sind. Diese Obergrenze läßt sich überdies 
angesichts der prinzipiell beacturänkten offentichen Mituel praktisch nich realisieren. Deshalb sind hier Allokutionsentscheidungen unter Knappheit unverzichtbar, für die allendings theoretisch voll befriedigende Verfahrea bisher nicht zur Verfügung stehen. Sowohl die verschiedenen Formen partizipatorischer Planung als auch die häufiger praktizierten Formen der Politikverflechtung und korportistiacher Verhandlungssysteme und erst recht die in der Bundesrepublik kaum praktizierten Formen zentralistischer Allokationsentscheidungen vermögen in steverungatheoretischer Hiasich
nicht voll zu befriedigen.

Welche Bedeutung haben nun rechtliche Regelungen in dem hier natürlich nur grob zu skizzierenden Zusammenhang? Zunächst düfte deutlich geworden sein, daß die eigentlichen Probleme dieser Interventionsform im Bereich der Allokationsentscheidungen liegen, also dessen, was gemeinhin auch mit dem Stichwort 'Planung' angesprochen wird. Was die Seite der Wirkungen auf die Adressaten angeht, so handelt es sich entweder um individuelle Nutzen, die sich aus der Inanspruchnahme der Einrichtungen unmittelbar ergeben und in ähnnutzung, deren Gewährleistung Soweit darüber hinaus jedoch Wirk der ökonomischen Interventionsform ist. Kompetenzen beabsichtigt sind, werden wir darauf im pädagogischen Interventionsform eingehen.

$\mathrm{Da}$ die in Frage stehenden Allokationsentscheidungen unter der Prämisse knapper Mittel getroffen werden müssen, sind hier Prozesse der Interessenartikulation und des Interessenausgleichs zwischen den beteiligten, im Regelfall hoch organisierten (staatlichen, parastaatlichen und privaten) Akteuren unverden unter sozialstaatlichen Gesichtspunkten interessierenden Verbesserung und des Ausgleichs von Teilhabechancen spielen hier regelmäßig machtpolitische Gesichtspunkte, spezifische Organisationsinteressen und gelenotwendigen Informationsdefizits und eines virtuellen Legitimationsdefizits Kooperation der 'bürgernäheren' Franz, Rosewitz, Wolf 1986: $543-552$ ) und ihre Verbände angewiesen (vgl. typischerweise nur eine untergeordnete Rolle, dagegen spielt deshalb hier und Partizipationsverfahren sowie Planungszwecke lassen sich Planungsregeln, auch wenn dies aufgrund politischer Widere grundsätzlich rechtlich

In steuerungstheoretischer Perspektive Widerstände häufig nicht geschieht. zung in der Weise zu wünschen, daß rung, sondern auch solche der Handlungt nur Prozesse der Handlungsnormieder Perspektive 'reiner Steuerungsformen' sind Vertchergestellt werden. In 
durch das Vorherrschen von Kollegialität gekennzeichnet: D.h. wemn überhaupt Prozesse der Handlungsnormierung und Handlungsbeurteilung (und nicht der bloben Interessenabstimmung im Rahmen von Tauschprozessen) stattfinden, so erfolgt Handlungsnormierung und Handlungsbeurteilung in der Form von Diskussions- und Überzeugungsprozessen (vgl. Majone 1986), wobei als mögliche Sanktionen die Zuweisung und Aberkennung von Achtung bzw. sozialem Einfluß fungieren. Derartige Prozesse sind in hohem Maße personenbezogen, fungieren also nur insoweit, als die Repräsentanten der verschiedenen Organisationen nicht beliebig austauschbar sind. Der in die gleiche Richtung wirkende, jedoch inhaltlich 'stärkere' Koordinationsmechanismus der Professionalitäl läbt sich in den hier in Frage stehenden Bereichen beim gegenwärtigen Stand unserer wissenschaftlichen Kenntnis über Wirkungszusammenhänge noch kaum einsetzen. Offen und unter dem Gesichtspunkt 'partizipatorischer Planung' viel diskutiert ist die Frage, inwieweit auch demokratische Steuerungsformen im Rahmen von Planungsprozessen mōglich sind. Sie scheitern wohl weniger an sachimmanenten Gründen dean an den Machtinteressen der beteiligten organisierten Akteure. Inwieweit ihnen allerdings nicht nur eine legitimierende, sondern auch eine evaluierende und damit effektivierende Funktion zukommen kőnnte, scheint offen.

\subsection{Padagogische Interventionsform}

Die Entwicklung sozialer Kompetenzen oder von 'Humanvermögen' ist nicht nur unter normativen Gesichtspunkten der Sozialstaatlichkeit, sondern auch unter Effektivitätsgesichtspunkten gesellschaftlicher Entwicklung zu begründen. Dieser zweite Gesichtspunkt ist jedoch bisher noch kaum ausgearbeitet, wenngleich plausibel: Wie sollte die heutige wirtschaftliche Leistungsfähigkeit bei einem durchschnittlichen Gesundheitszustand und Bildungsgrad wie vor hundert Jahren gewährleistet werden? Die Ansprüche, die das moderne Leben an die Aufmerksamkeit, Zuverlässigkeit, Anpassungsfähigkeit, Leistungsbereitschaft, Frustrationstoleranz, Differenzierungsvermögen und Wissen der Bevölkerung stellt, gehen weit über das in der bisherigen Menschheitsgeschichte Notwendige hinaus - oder sind zum mindesten in der Art völlig anders und auf eben die spezifischen Leistungen angewiesen, die durch die verschiedenen Formen der Sozialisation, Bildung, Rehabilitation und Beratung erst ermöglicht werden.

Ein erheblicher, wenngleich im historischen Vergleich vermutlich sinkender Teil dieser Leistungen wird sozusagen 'naturwüchsig' im Rahmen von Familie, Nachbarschaft, Vereinigungen und sonstigen Solidaritätsformen erbracht. Daneben sind jedoch in steigendem Umfange öffentliche Leistungen getreten die Einführung der allgemeinen Schulpflicht markiert hier die Schwelle zur wohlfahrtsstaatlichen Entwicklung am deutlichsten. 
Die Systematisienung der hier ablaufenen Prozesse in steverungatheoretischer Perspektive steht noch in ihren Anfangen. Sicher ist, daß der Prozeß der Inanspruchnahme sozialer Dienste mit zahlreichen Hemmnissen und 'Filtern' belastet ist, die eine sozusagen gradlinige Realisierung entsprechender sozialstaatlicher Intentionen in Frage stellen (vgl. Wirth 1982). Allerdings hängt es in erheblichem Umfange von der Struktur der leistungserbringenden Einrichtungen wie auch den Handlungskompetenzen ihres 'Publikums' ab, ob und wie effektiv die hier notwendigen 'Koproduktionsprozesse' ablaufen (vgl. Wirth 1986b).

Entscheidend für diese Interventionsform ist somit der Umstand, daf ihre Wirksamkeit ganz entscheidend von der Endstufe des gesamten Prozesses, also der Interaktion zwischen den die Endleistung produzierenden Einrichtungen und deren Publika abhängig ist. Es kommt also entscheidend auf die Leistungsfahigkeit des im unmittelbaren Publikumskontakt stehenden Personals an, das innerhalb der in Frage stehenden Organisationen zumeist nur einen untergeordneten Status einnimmt (vgl. Grunow 1978). Während in all den Fällen, wo die $2 u$ vermittelnden Leistungen standardisierbar sind (2.B. Geldleistungen, Sachleistungen i.e.S.) eine konditionale Programmierung und damit weitgehende 'Bürokratisierung' der Leistungserbringung zwar mit gewissen Frustrationen für die Leistungsempfänger verbunden sein mag, aber doch im Kern die Wirksamkeit nicht beeinträchtigt, muß für unmittelbar personenbezogene Dienstleistungen eine allzu eingehende rechtliche Normierung der Leistungserbringungsprozesse als im strengen Sinne wirkungswidrig vermutet werden. Allerdings wäre auch diese Vermutung zu differenzieren: Ärztliche Leistungen lassen sich z.B. in hohem Umfange typisieren und im Rahmen von Gebuihrenordnungen bewerten. Die Kombination der Leistungen und die Beurteilung ihrer Notwendigkeit im Einzelfall läßt sich dagegen rechtlich nicht mehr regulieren. Die entsprechenden Kontrollversuche von Krankenkassen und kassenärztlichen Vereinigungen operieren mit Durchschnittswerten pro Arzt, deren Fragwürdigkeit offenkundig wird, sobald beispielsweise die Altersstruktur des Patientenstammes verschiedener Ärzte sehr unterschiedlich ist. Noch deutlicher haben sich die Versuche dig erwiesen, die curriculare Bewegung ist nach kurzer Blüte schnell an ihre organisatorischen, pädagogischen und politschen Grenzen gekommen.

Ein Beispiel erfolgreicher sozialpolitischer Reform stellt die Pädagogisierung des Elementarbereichs in den 70er Jahren dar (vgl. Domscheit, Kaufmann, Kühn 1980; Domscheit, Kühn 1984). Eine wesentliche Charakteristik dieser Reform bestand jedoch gerade darin, daß die zugrunde liegenden gesetzlichen Normienungen hier sehr offen blieben und lediglich die allgemeine Richtung einerseits die verstärkter Form regulativen Rechts erfolgte. Entscheidend war wesens (ökologische Intervention), ander und der Ausbau des Kindergarten- 
tionsniveaus des Kindergartenpersonals durch eine Verbesseung der Ausbildungseinrichtungen, die Anhebung der tariflichen Einstufung mit entsprechender Erhöhung der Qualifikationsanforderungen, und nicht zuletzt die Einrichtung eines Fachberatungssystems in enger Abstimmung mit den Trägerorganisationen (vgl. o. Fig. 2). Die Rekonstruktion des Reformverlaufs zeigt, daß dabei den (zum mindesten in Nordrhein-Westfalen dominierenden) Trägern der freien Wohlfahrtspflege entscheidender Einfluß zukam. Die Pointe dieses Erfolgs ist allerdings darin zu sehen, dal das Kindergartensystem als Ganzes in jener Zeit unter einen erheblichen Erfolgszwang gestellt war, da zahlreiche bildungspolitische Bemühungen dazu tendierten, die Vorschulerziehung dem Elementarbereich auszugliedern und dem Schulwesen anzugliedern.

Alles in allem sind also die Steuerungsleistungen des Rechts im Rahmen der pädagogischen Interventionsformen sehr beschränkt. Es kann hier lediglich darum gehen, Wirkungselemente anderer Steuerungsformen (Kollegialität, Professionalität, Solidarität) zur Geltung zu bringen und Vorkehrungen zu treffen, daß die diesen Steuerungsformen immanent partikularistischen Züge hinsichtlich ihrer Auswirkungen in Grenzen gehalten werden.

\section{Zusammenfassung}

Die Verwirklichung sozial- bzw. wohlfahrtsstaatlicher Zielsetzungen ist auf das Recht als das charakteristische Organisationsmittel des Staates mit Notwendigkeit angewiesen. Es gibt jedoch gute Gründe für die Annahme immanenter Grenzen der Wirksamkeit rechtlicher Steuerung im Wirkungsfeld wohlfahrtsstaatlicher Zielsetzungen. Sie genauer zu bestimmen war die Absicht der vorangehenden Überlegungen.

In dem Maße, als das Recht von einer 'moralgetränkten Ordnung' zu einem positivierten Steuerungsmittel des Staates wird, sinkt seine soziale Verbindlichkeit. Die 'Verrechtlichung' im skizzierten fünffachen Sinne des Wortes treibt somit gleichzeitig die Einwirkungsmöglichkeiten des Staates voran und beeinträchtigt ihre soziale Wirksamkeit.

Dabei stößt die rechtsformige Gestaltungsabsicht des Staates auf eine doppelte Grenze: Zum einen auf diejenige entgegenstehender Interessen, die sich mit mehr oder weniger Erfolg um so eher einem mißliebigen Regulierungsanspruch zu entziehen suchen, als die moralische Verbindlichkeit des Rechts sinkt. Hier gibt es unter rechtsstaatlichen Gesichtspunkten keine Alternative zu einer weiteren 'Verrechtlichung', die ihre Grenzen im wesentlichen an der Steigerung des Kontroll- bzw. Durchsetzungs- und Judizialisierungsaufwands, oder in ökonomischer Formulierung: der Transaktionskosten, findet. Eine zweite Grenze ergibt sich aus dem spezifischen Kriterium sozialstaatlicher Intervention, der es um die Ermöglichung sozialer Teilhabe auch für sozial 
benachteiligte Bevollkerangsgruppen geht. Then ist die verrechtichte Kommunjkationsform weitgehend fremd, und so encheinen sie zwar als Rechtertigungsgrund, oft aber nicht als die tatslichlich Beglnatigten wohlfahrtsataulicher Maßahmen. Auch diese Aussage ist allendings nach dem Typus der sozialpolitischen Interventionen $\mathrm{zu}$ differenzieren: Wathrend bei der rechulichen und ökonomischen Interventionsform der rechusformigen Steuerung zentrale Bedeutung zukommt, ist ihre Wirkungsmoglichkeit bei der Okologischen und der pädagogischen Interventionsform aus unterschiedlichen Gründen sehr beschrünkt.

Insoweit als die Gesetzgebungstheorie die mogliche Wirksamkeit staatlicher Mafnahmen als Beurteilungskriterium anertennt, ist aus sozialwiscenschaftlichen Untersuchungen zur Wirkungsweise staatlicher Interventionen grundsäzlich brauchbares Wissen zu erwarten. Dieses wird allerdings häufig nur politikbereichsspezifisch aufbereitet werden können. Theorien mittlerer Reichweite sind rechtspolitisch informativer als allzu generelle soziologische Theorien. Damit sind allerdings auch einer allgemeinen Gesetzgebungstheorie inhattliche Grenzen gesetzt. 'Rechtspolitische Rationalisierungsgewinne' sind wahrscheinlich eher im Rahmen bereichsspezifischer Fachöffentlichkeiten zu erzielen als auf dem Niveau einer allgemeinen Gesetzgebungslehre.

\section{Anmerkungen}

1 Girvetz (1968: 512) definiert mit wünschenswerter Klarheit: "The Welfare State is the institutional outcome of the assumption by a society of legal and therefore formal and

2 Hierzu ausfithichily for the basic well-being of all of its members."

3 Von besonderer Ber Kaufmann 1985b, 1986a.

deutschen Staatsrechteutung in diesem Zusammenhang die Beratungen der Vereinigung der staat" und die anschlier auf der Jahrestagung 1971 über "Grundreche im LeistungsZusammenfassend Grimm 1990 Diskussion. Vgl. Martens 1972 und Hzberle 1972. Zacher 1980.

Forstorf 1968 und eingehend lichen auch auf weiten Bedeutungsraums des Begriffs 'Wohlfahrt' besteht im Grundestzlich der wichtigaten Dimensior Ebene eine überraschend hohe Übereinatimmung hineichAufgabe staatlichen Handelns sein menschlicher Lebensbedingungen, deren Gewăhrleistung der allgemeinen Erklărung der Menschenrechrammatische Formulierungen finden sich in in verbindlicherer Form in der Europarischente der Vereinten Nationen (Ar. 22-27) und ischen Sozialcharta. Auch die Diskussion Menschenrecheskonvention und der Europăkonsensfahige Bereiche wohlfahrtetsastion um soziale Indikatoren hat international 1973. - Zacher (1984: $46 \mathrm{ff}$.) betont, doß Zieluetzungen erkennen lawen, vgi. OECD (scil. des Verfassungs- und internationalen "hinter dieser Schwliche vorordnenden Rechss nicht vergessen werden darf, daß Sozialpo Rechts, F.X.K.) gegenüber dem Sozialrecht

5 Genauere Vergleichsstudien $57 \mathrm{f}$. 
6 Wir benirzen den Terminus 'Scainlpolitik' im folgenden nicht in instintionellen Sinne, soadern ts analytischen Begriff zur Kennzeichnung antlich induzierter Maßnahmea, die auf die Beeinfluseung sozialer Teilhabemöglichkeiten gerichtet sind. Hierzu susführlicher Kaumann 1982a: 55-63.

7 Wie die Geachiche der Soxialpolitik zeigt, orientient sich die Gesetzgebung hilufig an exemplariachen Problembaungen, die zuvor auf kommunaler, genoseenschaftlicher oder freigemeinnilziger Ebene erprobt wurden, z.B. Krankenversicherung, Sozialer Wohnungsbav. Wo der Stant bereit existienende Triger bericksichtigt, erfolgt die stantiche Intervention meist inkremental i.S. fortachreitender Standardisierung und Vernetzung. Werden dagegen neve Einrichtungen von Stum wegen geschaffen (z.B. Arbeitsverwaltung), ist der Grad der Zentralisierung und Vereinheitlichung von Anbeginn hōher.

8 Zur merkantilistiechen Wohlfahrtupolitik vgl. Maier 1966.

9 Dies it des Thema der sog. Korporatimus-Diskussion, vgl. Kirberger 1978, Schmitter und Lehmbruch 1979, Alemnnn und Heinze 1979, Offe 1981.

10 Natirlich hat da Problem der Bewirkbarkeit sozialpolitisch intendierter Wirkungen noch einen anderen, spezifisch politischen Aspekt: Da Sozialpolitik ihrem normativen Gehalt nach stets den "Schutz des Schwibcheren" (hierzu v. Hippel 1982) intendiert, müseen ihre Forderungen grundutzlich eyen den Strom der gesellschaftichen Machtverballtnisee durchgesetzt werden. Die Frage, unter welchen Bedingungen sozialpolitische Gesetze bzw. Programme politich verwirtlickt werden kơnen, liegt auBerhalb des hier zu behandelnden Problemkreises.

11 Hierzu zuammenfasend Luhnans 1984: 51-70 und allgemeiner Maturana 1982: bes. 14-31, 170-225, sowie G. Teuber in dievem Band. Der Grundgedanke besagt in unserem Zusammenhang, daß jedes soziale System zu seiner Selbsterhatung auf bestimmte Konatitutionabedingungen angewiecen ist, deren Berücksichtigung sein Verhalten gegenüber Aufeneinflüsen nachhaltig bestimmt. 'Steuerung' i.S. von gezielter Verhaltensbeeinflussung eines Syatems durch ein anderes System erscheint von daher als grundsätzlich unwahracheinlich bzw. nur unter sehr spezifischen Bedingungen überhaupt möglich.

12 Die dabei relevanten Unterscheidungen können mit den rechtsdogmatischen Klassifikationen nur zufalligerweise äbereinstimmen, da eine der zentralen Aporien gegenwărtiger Rechtsdogmatik ja gerade in der Nich-Integrierbarkeit prospektiver Folgenbetrachtung liegt. Vgl. Teubner 1984: $309 \mathrm{f}$.

13 Die Differenzen zwischen der kontinemaleuroptischen und der angelsăchsischen Staatsentwicklung werden bei Habermns wie bei den meisten deutschen Theoretisierungsversuchen politischer Modernisierung zu wenig berücksichtigt. Aus demselben Grunde ist es fragwürdig, die deutache 'Verrechtlichungadebatte' allzusehr mit der US-amerikanischen Diskussion über 'Deregulation' zu parallelisieren. Letztere ist eher mit der in der Bundesrepublik geführen 'Privatisierungadebatte' zu vergleichen.

14 Luhmann (1981: 95) weint darauf hin, daß "mit Hilfe von Rechtssetzung und Geldbewilligung in der Zentrale schon politische Erfolge erreicht werden - und dies ohne Rücksicht auf die faktischeo Auswirkungen solcher Maßnahmen". Das Faktum des zum mindesten vorübergehenden politischen Erfolges von 'Wahlgeschenken', 'symbolischem Politikgebrauch' oder auch wohlmeinendem Irrtum über Gesetzesfolgen ist kaum zu bestreiten. Auch bedarf eher aubergewobnlicher Umstinde, daß die Konsequenzen politischer Fehlentscheidungen diejenigen treffen, die sie tatshlchlich zu verantworten haben. Auch wenn sich solche Intransparenz infolge der notwendigen Komplexităt der Verhsiltnisse nicha grundsătzlich beseitigen 1 $1 \mathrm{ABt}$, bleibt der Anspruch auf Wirksamkeit notwendiges Element einer normativen Begrundung von Politik (vgl. den Beitrag von Zeh in diesem Band). Die Gesetzgebungslehre und die wissenschaftliche Analyse einzelner Sachpolitiken kơnnen dazu beitragen, solche Begründungsprozesse zu versachlichen. 


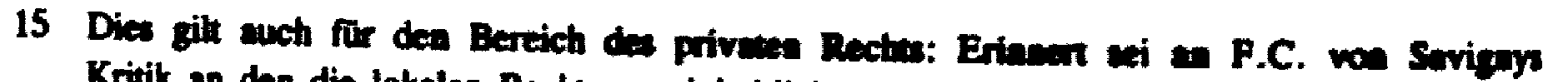

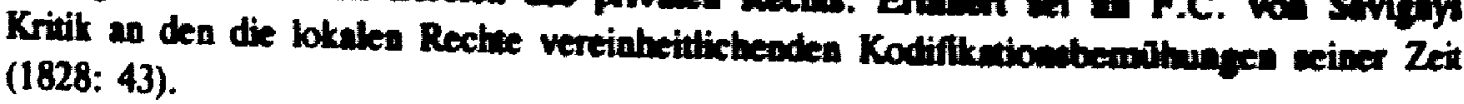

16 Der Begriff der Bürokratisierung deckt in historischer und altweller Perspektive eine Vielzahl interdependenter Prozese auf allen Ebenen socialer Wirtlichkeit, vgl. Grunow

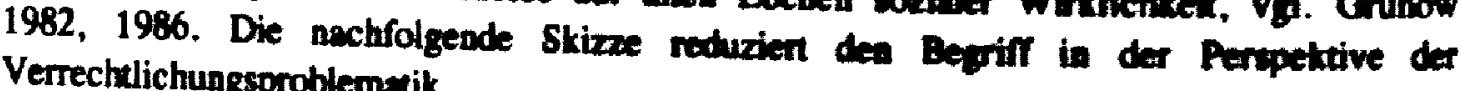

17 Nur am Rande sei angemerkt, das die Dimewionienung sich waf ein Mehrebenenmodell soziologischer Analyse bezieh: Der Begriff der Verwamlichung iu der Gesellactuftuebene, derjenige der Vergesetzlichung der institutionellen Ebene, derjenige der Börokrativierung der organisatorischen und interorganiestoriachen, dejendfe der Verrectulichung coxialer Beziehungen der interaktiven Ebene zuzuordnen. Zur Kowophulliterung diever Bbenew vgl. Kaufmann 1982b: 256-260; Grunow 1982: 158-218.

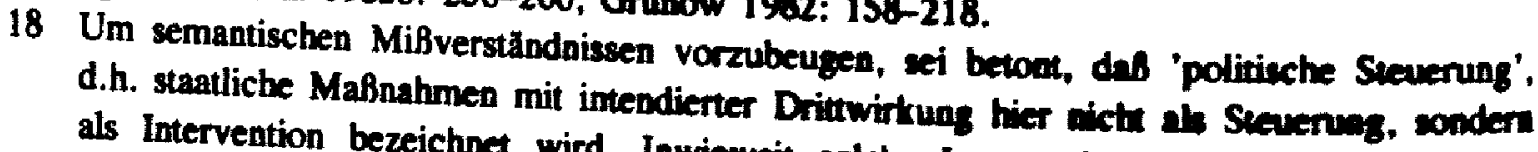
als Intervention bezeichnet wird. Inwieweit solche Intervewionea Stevenungowirkungen entfalten, ist ja gerade das Problem!

19 So explizit der Ansatz der interdisziplinaren Forschungerouppe "Steuerung und Erfolgakontrolle im offentlichen Sektor" (Kaufmann, Majone, Otrom (ed.) 1986) aber woch tie von Teubner (1984) und Willke (1983, 1984) entwickelten Perspektiven zeigen ahaliche

Im Rahmen der Diskussionen auf der diesen Bande zugrunde liegendea Tagung nahn die Ronfrontation dieses Ansatzes mit demjenigen von G. Teubner breiten Raum ein. Um den theoretischen Ausganges nicht zu sprengen, mus auf eine ausfubrliche Pribentition der Kaufmann $1986 \mathrm{~b}$. Im Unterachied and Grundbegriffe hier verzichtet werden, vgl. hierzu der hier vertretenen Steuerungstheorie Theorie aupoletischer Syuteme handelt es sich bei Sozialen, sondern un eine Theorie nicht un eine allgemeine Kontitutionatheorie ds Akteuren, die man sich Den theoretischen Ausgangspunk autopoietische Systeme vorstellen kann, erkitiren will. nicht nur Umweltoffenheit!) personaler und die Annahme der Umweltabhingigkeit (und von 'bounded rationality' nảher bestimmte) sozialer Syateme. Deren (durch die Annahmen ten bestimmen den Focus der Steverunge) Aktorqualităt und nicht ihre Systemeigenschafden Systemeigenschaften von Peruerungstheorie. Erst in zweiter Linie ist sodann zwischen

21 Diese Aussage gilt in dem Mersonen und Organiutionen als Akteuren zu unterncheiden. der Positivierung des Rechts in als Recht und Moral auneinandertreten, wie es als Folge 1985a. Kurzbezeichnusfuhrlich Kaufmann 1986b; leider kann eine Uberzeugende deutsche genden $Z$ wecke übersetzen wir Guionen noch nicht vorgelegt werden. Für die nachfollungskontrolle und Evaluation mit Handlungit Handlunganormierung, Control mit Hand-

23 Sotschsprachigen Begrifflichkeit muß einer späteren Bub; die genauere Ausarbeitung der So mag beispielsweise der Umstand späteren Publikation vorbehalten bleiben. verboten sind, ihre Wertigkeit für das Indiviturimmte Verhaltensweisen geboten oder

24 Fristsetzungen andere Prioritžtten überlagern. an bestimmte Adressaten richten "unoponlich formulierte Verhaltensvorschriften ..., die sich Gebote, Verbote oder bedingte Erlaubnisse formuliert seionsdrohung verknüpft sind und als

25 Dezentrale Kontextstewerung: "Politische formuliert sein können" (Mayntz 1983: 51). Interaktionsbeziehungen verabschieden Steverung muß sich vom Leitbild hierarchischer 


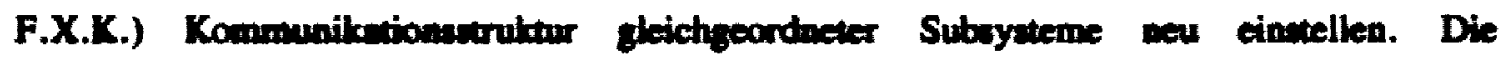
Bedingung der Notwendigkeit politiecher Steuerung - die Autonomie der Teile - muß zugleich als Bedingung der Moglichkeit der Intervention begriffen werden: Steuerungsimpule sind móglich in der Form der Konditionalisierung der Randbedingungen, die als beobachabere Differenzen die Informationabasis der jeweiligen besalen Zirkularität (richtiger: der Akteure, F.X.K.) abgeben" (Willke 1984: 48). Zur Steverungsproblematik korporatitiacher Handlungasyzeme vgl. Teubner 1979, Gouch 1984; Schimank/Glagow (1984) weisea jedoch zu Rech darau hin, dab nich alle Formen stanticher Einbeziehung von Verbundshandeln uner den Begriff des Korporatiumus subsumiert werden sollten.

26 Betrachtet man die bistorische Entwicklung der wohlfahtsutantichen Interventionen, so zeif sich, dab der erfolgreiche Aufbu von Politikbereichen durch eine allmăhliche 'Verrechlichung' (im oben beschriebenen fünffachen Sinne des Wortes) gekennzeichnet int. Etablierte Politikbereiche sind zudem durch eine Fachöfentlichkeit gekennzeichnet, im Rahmen derer epezialivierte Meinungsbildungrprozene zwischen Wisenschaftern, Verwaltungsleuten, Politikern und Fechjournalisten stattfiaden. Auch wenn derartige Meinungabildungaprozese gegen Imimer nich gefeit sind, ist von ihnen im Laufe der Jahre doch ein bemerkenswerner Beitrag aur kognitiven Aufhellung der Wirkungsbedingungen innerhalb eines betimmen Politikbereichs zu erwarten. Zur Bedeutung derartiger 'Policy Communities' vgl. Shere 1986, Majose 1986.

27 "Wean man als Reche das bezeichnet, was verhaltenenisig in Erscheinung tritt, so wird es in Organisationen formuliert, $*$ wird weitgehend von Organisationen überwach und durchgefohrt, und $\&$ richtet sich uberwiegend an Organisationen als Adressaten seiner Norm. ... Diese Organisationsgebundenheit (spielt) in Selbstventăndnis und in den Theorien der Rechtswiseenschaftler nur eine untergeordnete Rolle ...: Weitgehead formulieren Rechtswissenschaftler ihre Begriffe an einem Modell von Rech, das die Steverung des Verhaltens von Individuen und die Regelung von Konflikten zwischen Pernonen in den Vordergrund rilck" (Blankenburg und Lenk 1980: 7).

28 Empirische Belege z.B. bei Kaupen 1972; zur objektiven Benachteiligung der Personengruppen mit geringer Organisationserfahrung im Lohnsteuerverfahren vgl. Linder 1972.

29 Nach unseren eigenen Untersuchungen scheint die Bereitschaft der Steuerzahler, ihre eigenen Interessen wahrzunehmen, stärker von der Einschătzung ihres Rechtswissens als von der tatsåchlichen Richtigkeit dieses Wissens abzuhăngen. Die Bereitschaft, sich sachverstăndigen Rats zu versichern, steigt überdies mit dem Bildungsgrad und der allgemeinen Erfahrung im Umgang mit Behörden. Vgl. Grunow, Hegner, Kaufmann 1978.

30 Einen breiten Überblick über die neueren Tendenzen zur Verrechtlichung im Bereich der Sozial- und Wirtschaftspolitik geben die Beiträge von H.F. Zacher, S. Simitis, F. Kübler und K. Hopt in Kübler 1984.

31 Angesichts der Vieldimensionalităt der Zusammenhănge sind Vereinfachungen unvermeidlich. Eine komplemeatäre, an den Phasen des politischen Prozesses und den beteiligten Akteuren orientierte Darstellung gibt R. Mayntz 1980: 238.

32 Vgl. Kaufmann 1982a: 66-86; zur Begründung dieses Ansatzes und zur Beurteilung anderer Klassifikationsvorschläge vgl. Kaufmann/Rosewitz 1983.

33 Zur Inklusion als gesellschaftstheoretischem Korrelat wohlfahrtsstaatlicher Zielsetzungen vgl. Luhmann 1981: 25 ff.

34 Die hier gemeinte Intention laßt sich nicht immer aus den Gesetzesmotiven oder angegebenen Rechtszwecken ableiten, ist jedoch i.d.R. aus dem Gesamtkontext bestimmter Maßnahmen unschwer zu erschließen. Sozialpolitische Gesetze entfalten ihre Wirksamkeit i.d.R. in mehreren dieser Dimensionen. Der Vorteil dieser analytischen Unterscheidungen liegt in unserem Zusammenhang darin, dab sie die Bedingungen der Effektuierung rechtlicher Regelungen genauer zu differenzieren gestatten. 


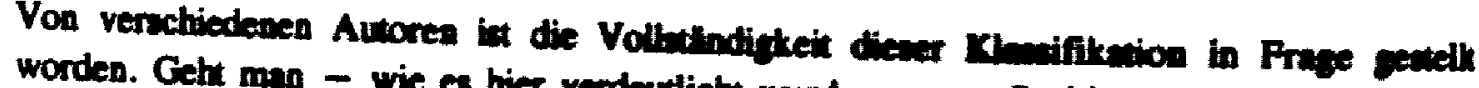
sozialatandicher Zielectie es hier verdeuticht munde - voen Problem der Verwirktichuns Bend. Ebenfalls auf die Lebentrectrite ich die Klasifitation anch wie vor fir abuchlie-

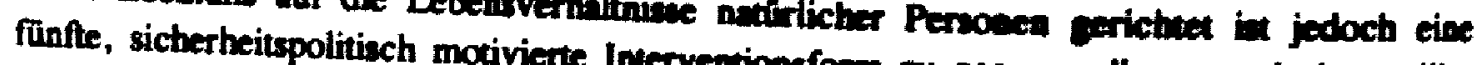

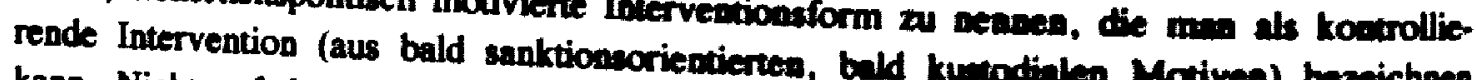
(ives, bald kustodialen Motiven) bezeichnes 167), die Einrichtung von Verhandlungar liegt der Vonchlas von Heinze/Olk (1994: betrachten. Verhandlungssysteme berietgayutemen als weivere Intervectionsform zu deutlich gemacht haben dürfte - guf den vorgech - wie die vorangehoude Dantellung allenfalls auf die Formen kollektiver Intereagelngerten verbandlichen oder Trigerbereich, sozialen Teilhabemöglichkeiten.

Im übrigen gelten hier die meisten Restriktionen, die in der Linermur zu dea Möglichkeiten und Grenzen regulativer Politik namhaft gemech werden (vol. etwa Noll 1972, Maynz
1979, 1983). Fürsorgepflicht ohne subjektive Rechene Mindeatlohngeactzgebung oder eine kommunale

38 Ein besflicht ohne subjektive Rechtsanspriche, wie sie vor dem BSHG bestand.

der ökonomischen Intervention Kombination unterschiedilcher Geldleistungen in Rahmen Regeln (2.B. Anrechnungsnormen), dar. Hierfür bestehen nur in Einzelfillea rechaliche uneinsichtig und 'unvernünftig' tionsmöglichkeiten, die aus sozialpinen. Umgekehrt ergeben sich aber auch KumulaProblem läft sich jedoch durch kolpolitischer Sicht 'perverse' Folgen zeigen. Dieses sondern nur durch politische und organisato Programmierung nich befriedigend lösen, der Sozialleistungen i.S. einer finalen Zielsetzung bedarfsadäquater Einkommensminimachungsweise, d.h. unter der expliziten

39 Hinzu kommt, daA puater Einkommensminima.

tern, Bildungs- und Beratungseinrichtungen ersen, wie sie in Krankenhalusern, TheaGüter sind, deren relativer Preis im Zuge der wirtschaftlichen Entwicklung enorm
gestiegen ist. Ohne entsprechende offentliche Subventisien einer konstanten, sondern mit einer bei gerechnet werden.

gedürnis rückläufigen Inanspruchnahme vermuten, daB hier steueruner Kompliziertheit noch wenig erforscht. Es ist allerdings $z u$ wăren, jedoch hăufig am Widerstandisch befriedigende Lơsungen grundsătzlich mơglich Akteure scheitern. Hierbei sind viand oder der fehlenden Einsicht besonders măchtiger bestehender Domånen ausgerichtete Organisationsinteressen Sicherung oder Erweiterung (vgl. Franz 1986: 483-492).

41 Man kann daraus den

Verhandlungssysteme nur unter externemgstheoretisch begründbaren Schluß zieben, daß sen gelangen.

\section{Literatur}

Alemann, U.v., Heinze, R.G. (Hg.), 1979: Verbände und Staat, Opladen gungen des Gesetzesvollzugs. Jahrbuch für Rechtseoziologist - Organisatorische Bedin-
Opladen 
Blankenburg, E., Treiber, H., 1962: Interpretationshernchaft îter die Grundreche als Kookurrenzproblem zwischen Rechn- und (empirisch-orientierten) Sozialwisenschaftlern. In: W. Hasemer u.A. (Hg.), Gundrechte und soziale Wirklichkeit, Baden-Baden, 9-37

Bockenforde, E.-W., 1976: Grundrechtstheorie und Grundrechtsinterpretation. In: Ders., Stan, Gesellachaft, Freiheit. Studien zur Staatstheoric und zum Verfassungsrecht, Frankfurt/M., 221-252

Bohae, E., 1980: Informales Verwaltungahandeln in Gesetzesvollzug. In: E. Blankenburg / K. Lenk, op.cit., 20-80

Domecheit, S., Kaufmann, F.X., Kahn, M., 1980: Innovative Politik im Bereich verbandlicher Wohlfahrtsplege am Beispiel der Kindergartenreform in Nordrhein-Westfalen. In: $R$. Mayntz (Hg.), op.cit., 176-199

Domscheit, S., Kühn, M., 1984: Die Kindergartenreform - Eine Pallstudie bundesdeutscher Sozialpolitik, Frankfurt/New York

Dyson, K., 1980: The State Tradition in Weatern Europe, Oxford

Elias, N., 1976: Uber den ProzeB der Zivilisation. Bd. 2: Wandlungen der Gesellschaft, Entwurf zu einer Theorie der Zivilistion, (zuerst 1939), Frankfurt/M.

Ellscheid, G., 1979: Die Verrechtlichung sozialer Beziehungen. In: Neue Hefte der Philosophie 17: Recht und Moral, Goutingen, 37-61

Forathoff, E., 1968: Rechusunutlichkeit und Sozialstantlichkeit, Darmstact

Franz, H.J., 1986: Interorganizational Arrangements and Coordination at the Policy Level. In: Kaufmann, Majone, Oatrom, op.cit., 479-494

Franz, H.J., Rosewitz, B., Wolf, H., 1986: Associations and Coordination. In: Kaufmann, Majone, Ostrom, op.cit., 531-555

Gessner, V., Winter, G., 1982: Rechesformen der Verflechtung von Staat und Wirtschaft, Opladen

Girvetz, H.K., 1968: Art. Welfare State. In: International Encyclopedia of the Social Sciences. Vol. 16: 512-521

Glagow, M. (Hg.), 1984: Gesellschaftssteuerung zwischen Korporatismus und Subsidiaritä, Bielefeld

Gotsch, W., 1984: Neokorporatismus in steuerungstheoretischer Perspektive. In: M. Glagow (Hg.), op.cit., 54-88

Gretuchmann, K., 1986: Solidarity and Markets. In: Kaufmann, Majone, Ostrom, op.cit., $387-405$

Grimm, D., 1982: Grundrechte und soziale Wirklichkeit - zum Problem eines interdisziplinăren Grundrechtsverstăndnisses. In: W. Hassemer u.a. (Hg.), Grundrechte und soziale Wirklichkeit, Baden-Baden, 39-76

Grimm, D., 1983: Die sozialgeschichtliche und verfassungsgeschichtliche Entwicklung zum Sozialstaat. In: P. Koslowski u.a. (Hg.), Chancen und Grenzen des Sozialstaats. Tübingen, 41-64

Grotrup, H., 1973: Die kommunale Leiatungsverwaltung - Grundlagen der gemeindlichen Daseinsvorsorge, Stuttgart

Grunow, D., 1978: Alltagskontakte mit der Verwaltung, Frankfurt/New York

Grunow, D., 1982: Bürokratisierung und Debürokratisierung im Wohlfahrtsstaat. Habilitationsschrift, Universităt Bielefeld

Grunow, D., 1986: Development of the Public Sector: Trends and Issues. In: Kaufmann, Majone, Ostrom, op.cit., 25-58

Grunow, D., Hegner, F., Kaufmann, F.X., 1978: Steuerzahler und Finanzamt, Frankfurt/New York

Habermas, J., 1981: Theorie des kommunikativen Handelns. Bd. 2: Zur Kritik der funktionalistischen Vernunf, Frankfurt/M. 
Hiberle, P., 1972: Grundrechte im Leistungatax, 2. Mitbericht. In: Veröfentlichungen der Vereinigung der deutschen Stantarechulehrer, Bd. 30, Berlia/New York, 43-141

Hegner, F., 1978: Das bürokratische Dilemma: $\mathrm{Zu}$ einigen untunbalichen Widerapruchen in den Beziehungen zwischen Organiaation, Perwoal und Publikum, Frnokfurt/New York

Hegner, F., 1986: Solidarity and Hierarchy: Institutional Arrangements for the Coondination of Actions. In: Kaufmann, Majone, Otrom, op.cit., 407-429

Heinze, R.G., Olk, T., 1984: Sozialpolitische Steverung: Von der Subridiaritit zum Korporetismus. In: M. Glagow (Hg.), op.cit., 162-194 431-443

Hippel, E.v., 1982: Der Schutz des Schwächeren, Tübingen

Hood, C., 1976: The Limits of Administration, London/New York

Hood, C., 1986a: The Hidden Public Sector: The 'Quangocratization' of the World. In: Kaufmann, Majone, Ostrom, op.cit., 183-207

Hood, C., 1986b: Concepts of Control over Public Bureaucracies: 'Comptrol' and 'Interpolable Balance'. In: Kaufmann, Majone, Outrom, op.cit., 765-783

Hucke, J., 1983: Regulative Politik. Das Beispiel Umweltschutz. In: R. Voigt, op.cit. (1983a), 50-66

Kaufmann, F.X. (Hg.), 1977: Bürgernahe Gestaltung der sozialen Umwelt - Probleme und theoretische Perspektiven eines Forschungsverbundes, Meisenheim

Kaufmann, F.X. (Hg.), 1979: Bürgernahe Sozialpolitik - Planung, Organization und Vermittlung sozialer Leistungen auf lokaler Ebene, Frankfurt/New York

Kaufmann, F.X., 1982a: Elemente einer soziologischen Theorie sozialpolitischer Intervention. In: Ders. (Hg.), Staatliche Sozialpolitik und Familie, München, 49-86

Kaufmann, F.X., 1982b: Art. Wirtschaftssoziologie, allgemeine. In: Handwörterbuch der Wirtschaftswissenschaften, Stuttgart etc., Bd. 9: 239-267

Kaufmann, F.X., 1984a: Was heißt Verrechtlichung und wo wird sie zum Problem. In: Ders. (Hg.), Ärztliches Handeln zwischen Paragraphen und Vertrauen, Düsseldorf, 9-23

Kaufmann, F.X., 1984b: Solidarităt als Steuerungsform. Erklärungsansătze bei Adam Smith. In: F.X. Kaufmann / H.G. Krüsselberg (Hg.), Markt, Staat und Solidarităt bei Adam Smith, Frankfurt/New York, 158-184

Kaufmann, F.X., 1985a: Rechtsgefüh, Verrechtlichung und Wandel des Rechts. Lampe (Hg.), Das sogenannte Rechtsgefühl. rie, Bd. 10, Opladen, 185-199

Kaufmann, F.X., 1985b: Major Problems of the Welfare State: Defining the Issues. In: S.N.
Eisenstadt / O. Ahimeir (Eds.). The Welfare State and

Kaufmann, F.X., 1986a: The Blurring Welfare State and its Aftermath, London, 44-56

Practice of the Welfare State. In

Kaufmann, F.X., 1986b: The Relationfmann, Majone, Ostrom, op.cit., 127-138 Kaufmann, Majone, Ostrom, op.cit.,

Subsidiaritat - Leitidee fir

Kaufmann, F.X., Rosewitz, Bur 1983

In: R. Maynt2 ( $\mathrm{Hg}_{\mathrm{g}}$ ), op.cit., 25-49

Kaufmann FX (H)

Bevollkenungsforschung und., 1984: Studien zum Drei-Generationenvertrag. Institut für

Kaurmann, F.X., Majone, G., Otrom, V. Materialien Bd. 15, Bielefeld

the Public Sector. The Bielefeld Interiscips.), 1986: Guidance, Control and Evaluation in 
Kaupen, W., 1972: Das Verhiltnis der Bevolkerung zur Rechtspflege - Empirische Mwerislien zur Frage der Effektivităt von Recht. In: M. Rehbinder / H. Schelsky (Hg.), Zur Effeltivitit des Rechs. Jahrbuch fur Rechtssoziologie und Rechtstheorie, Bd. 3, Disseldorf, 555-563

Kirberger, W., 1978: Staatsentlastung durch private Verbände, Baden-Baden

Krüsselberg, H.G., 1977: Die vermðgenstheoretische Dimension in der Theorie der Sozialpolitik. In: C.v. Ferber / F.X. Kaufmann (Hg.), Soziologie und Sozialpolitik. Sonderheft 19 der Kolner Zeitechrift für Soziologie und Sozialpolitik, Opladen, 232-259

Krüseelberg, H.G., 1985: Markets and Hierarchies. In: Kaufmann, Majone, Ostrom, op. cit., 349-386

Kübler, F. (Hg.), 1984: Verrechtlichung von Wirtschaft, Arbeit und sozialer Solidarităt Vergleichende Analysen, Baden-Baden

Lehner, F., 1979. Politikverflechnung: Institutionelle Eigendynamik und politische Kontrolle. In: J. Matthes (Hg.), Sozialer Wandel in Westeuropa. Verhandlungen des 19. Deutschen Soziologentages, Frankfurt/M., 611-627

Leibfried, S., Tennstedt, F. (Hg.), 1985: Politik der Armut und die Spaltung des Sozialataats, Frankfurt/M.

Linder, H., 1972: Die Inanspruchnahme steverlicher und vermogenspolitischer Vergünstigungen durch die Lohnsteuerpflichtigen, Opladen

Luhmann, N., 1972: Rechtssoziologie $1+2$, Reinbek

Luhmann, N., 1981: Politische Theorie im Wohlfahrtsstaat, München/Wien

Luhmann, N., 1984: Soziale Systeme: GrundriB einer allgemeinen Theorie, Frankfurt/M.

Maier, H., 1966: Die ältere deutsche Staats- und Verwaltungslehre (Polizeywissenschaft), Neuwied/Berlin

Majone, G., 1986: Mutual Adjustment by Debate and Persuasion. In: Kaufmann, Majone, Ostrom, op.cit., 445-458

Martens, W., 1972: Grundrechte im Leistungsstaat, 1. Bericht. In: Veroffentlichungen der Vereinigung der deutschen Stantsrechaslehrer, Bd. 30, Berlin/New York, 7-42

Maturana, H.R., 1982: Erkennen: Die Organisation und Verkorperung von Wirklichkeit, Braunschweig/Wiesbaden

Mayntz, R., 1979: Regulative Politik in der Krise? In: J. Matthes ( $\mathrm{Hg}$.$) , Sozialer Wandel in$ Westeuropa. Verhandlungen des 19. Deutschen Soziologentages, Frankfurt/M., 55-81

Mayntz, R. (Hg.), 1980: Implementation politischer Programme - Empirische Forschungsberichte, Königstein/Ts.

Mayntz, R. (Hg.), 1983: Implementation politischer Programme II: Ansātze zur Theoriebildung, Opladen

Mayntz, R., 1983a: Implementation von regulativer Politik. In: Dies., op.cit., 50-74

Nettl, J.P., 1968: The State as a Conceptual Variable. In: World Politics 20: 559-592

Noll, P., 1973: Gesetzgebungsiehre, Reinbek

OECD, 1973: List of Social Concerns Common to Most OECD Countries, Paris

Offe, C., 1981: The Attribution of Public Status to Interest Groups: Observations on the West German Case. In: S. Berger $(\mathrm{Hg}$.$) , Organizing Interests in Western Europe: Pluralism,$ Corporatism, and the Transformation of Politics, Cambridge, 123-158

Ostrom, E., 1986: A Method of Institutional Analysis. In: Kaufmann, Majone, Ostrom, op.cit., 459-475

Pressman, J., Wildaysky, A., 1973: Implementation, Berkeley

Savigny, F.C., 1828: Vom Beruf unserer Zeit für Gesetzgebung und Rechtswissenschaft, 2. Aufl., Heidelberg

Sharpe, J., 1986: Intergovernmental Policy-Making: The Limits of Subnational Autonomy. In: Kaufmann, Majone, Ostrom, op.cit., 59-81 


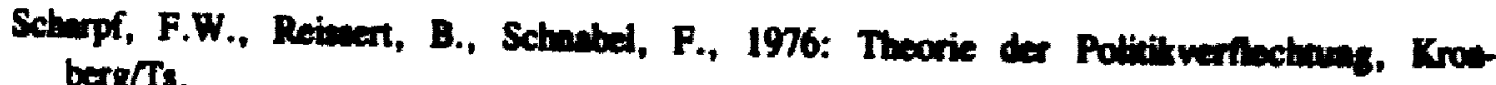
berg/ts.

Scharpf, F.W., Reiswen, B., Schnabel, F., 1978: Policy Effectivenes and Coaflica Avoldance in Intergovernmental Policy Formaion. In: Heof, K., Scherp, F.W. (Hg.), Introrganizational Policy Making, Loadoa/Beverly Hill, 57-115

Schimank, U., Glagow, M., 1984: Formen politiveher Steverung: Etcionne, Subsidierith, Delegation und Neokorporatiamus. In: M. Glagow, op.cil., 4-28 Schmitter, P.C., Lehmbruch, G. (H.), 1979: Trends Townds Capontin Incruediation,
London

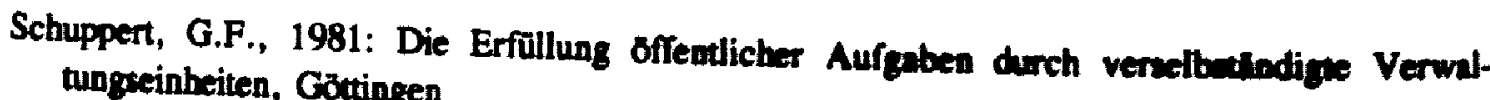

Teubner, G. 1979: Verrechtlichung der Arbeitubeziebungen. In: F. Kobler, op.cit, 73-165 Statliche Strukturvorga-Korporatistische Stratepien rechulicher Organiantionateverung:

Zeitschrift für Parlamentsfragen 10: 487-502

(Hg.), op.cit., 289-344

Voigt, R. (Hg.), 1980: Verrechtlichung, Konigatein/Ts.

Voigt, R., 1981: Mehr Gerechtigkeit durch mets Gesetz? In: Aus Politik und Zeitgeschichte.

Beilage zur Wochenzeitung 'Das Parlament', B 21
Voigt, R. (Hg.), 1983a: Abschied vom Recht, FrankfurtM.
Voigt, R. (Hg.), 1983b: Gegentende

Voigt, R. ( $\mathrm{H}_{\text {g.) }}$ 1983b: Gegentendenzen zur Verrechlichung. Jahrbuch fur Rechweoziologie und Rechtstheorie. Bd. 9, Opladen

Willke, H., 1983: Entzauberung des Stastes. Überkgungen zu einer wozialen Steverungaheorie, Königstein/Ts.

Willke, H., 1984: Gesellschaftssteuerung. In: M. Glagow, op.cit., 29-53 New York

Wirth, W., 1986a: Control in Public Administration: Plurality, Selectivity and Recundancy. In: Kaufmann, Majone, Oatrom, op.cit., 595-624

Wirth, W., 1986b: Public Administration

Affected Citizens. In: Kaufmatration and Publics: Control of Bureaucratic Performance by

Wulfhorst, S.T., 1982: Leistungan, Majone, Ostrom, op.cit., 739-763

Vierteljahresschrift für Sozigateigerndes Verhalten der Berechtigten in Sozialrech. In:

10, 1-18

Deutschland, Berlin 73-165 